\title{
An integrated strategy of MS-network-based offline 2DLC-QTOF-MS/MS coupled with UHPLC-QTRAP'-MS/MS for the characterization and quantification of the non-polysaccharides in Sijunzi decoction
}

\author{
Bangjian Dong ${ }^{1} \cdot$ Chongsheng Peng ${ }^{1} \cdot$ Ping Ma $^{1} \cdot$ Xiaobo $\mathrm{Li}^{1}$ \\ Received: 4 January 2021 / Revised: 17 February 2021 / Accepted: 18 March 2021 / Published online: 13 April 2021 \\ (C) Springer-Verlag GmbH Germany, part of Springer Nature 2021
}

\begin{abstract}
Sijunzi decoction (SJZD), a classic recipe in traditional Chinese medicine (TCM), has been applied for the clinical treatment of gastrointestinal diseases. While there are reports on pharmaceutical substances of SJZD focusing on its polysaccharides, the composition of non-polysaccharides (NPSs) has not yet been holistically clarified. In the current study, offline two-dimensional liquid chromatography coupled with quadrupole time-of-flight tandem mass spectrometry (2DLC-QTOF-MS/MS) was used for comprehensive NPS chemical profiling of SJZD. In addition, the MS-network of SJZD was proposed, which led to the construction of a larger in-house chemical library and accelerated qualitative processing. Four hundred forty-nine components, among which 6 were potentially novel, and 32 were confirmed by standard substances, were identified or tentatively assigned. Furthermore, based on good method validation, 19 representative components were simultaneously quantified by ultra-highperformance liquid chromatography coupled with triple-quadrupole linear ion-trap tandem mass spectrometry (UHPLCQTRAP $^{\circledR}$-MS/MS). They were selected for quantification on the account of their bioactive reports on in vivo or in vitro activities, the peak intensity in the mass spectrum, and characteristic structures, which have the potential to be qualitative or quantitative markers of SJZD. The present work furthers understanding of the pharmacological effects and action mechanism of NPSs in SJZD, and provides a useful analytical approach for complex composition research of TCMs.
\end{abstract}

Keywords Sijunzi decoction - Chemical profiling - Secondary metabolites - 2DLC-QTOF-MS · MS-network · UHPLC-QTRAP ${ }^{\circledR}$-MS

\section{Introduction}

Traditional Chinese medicine (TCM) formulae are being increasingly used in China and other Asian countries for their broad effectiveness and low toxicity. Several TCM formulae exhibited novel clinical effects, thus attracting the interest of many scientists. For example, Qingfei Paidu decoction and Lianhua Qingwen capsule have been included in the 6th-8th edition of the official guidelines as the primarily

$\overline{\text { Bangjian Dong and Chongsheng Peng contributed equally to this work. }}$

Xiaobo Li

xbli@sjtu.edu.cn

1 School of Pharmacy, Shanghai Jiao Tong University, No. 800 Dongchuan Road, Minhang District, Shanghai 200240, China recommended formulae for treatment of COVID-19 in China due to their obvious benefit to COVID-19 patients [1]. As is well-known, TCMs are featured as having "multicomponent," "multi-target," and "synergistic therapeutic values," thus indicating their working mechanisms are closely and directly attributed to complex ingredients. Therefore, a comprehensively qualitative and quantitative analysis of the chemical substances of TCM formulae constitutes the substantial basis for the further elucidation of its bioactive components and pharmacological mechanism.

Sijunzi decoction (SJZD), as a classic recipe in TCM formulae used to strengthen the spleen and replenish Qi, consists of Ginseng Radix et Rhizoma, Atractylodes Macrocephalae Rhizoma, Poria, and Glycyrrhizae Radix et Rhizoma combined in a 3:3:3:2 mass ratio. Pharmacological studies have revealed that SJZD displays extensive bioactivities such as 
gastrointestinal-regulating [2-4], antioxidant [5, 6], immunoregulatory $[7,8]$, and anti-tumor activities $[9,10]$. Meanwhile, both polysaccharides and non-polysaccharides (NPSs), which mainly include flavonoids, saponins, and terpenoids, are considered two major constituents responsible for its pharmacological or therapeutic effects. Until now, the majority of these studies have focused on the isolation and pharmacological activity of its polysaccharides. For instance, in our previous report, the protective effects of three polysaccharide fractions (S-1, S-2, and S-3) obtained from SJZD were investigated on reserpine-induced Wistar rat models. The results revealed that fraction S-3 could ameliorate the symptom of spleen deficiency [11]. Then, fraction S-3 was further purified to acquire a homogeneous polysaccharide S-3-1 with a molecular mass of $13.5 \times 10^{4} \mathrm{Da}$, which exerted an immunomodulatory role by regulating gut microbes $[7,8]$. However, the NPSs in SJZD have not yet been comprehensively interpreted. To the best of our knowledge, there are only a few phytochemical reports on SJZD, regarding flavonoids, saponins, and terpenoids, which used conventional chromatographic techniques, such as liquid chromatography (LC) and liquid chromatography-mass spectrometry (LC-MS) [12-15]. Restricted by the limited resolving power of one-dimensional (1D) separation, the qualitative characterization of NPSs in SJZD is not adequate as it omits some components, especially the minor and the isomer ones. Besides, identifying minor constituents promotes the research of pharmacodynamic substances of SJZD and provides valuable information on lead compounds for drug development [16].

Nowadays, ultra-performance liquid chromatography coupled with quadrupole time-of-flight tandem mass spectrometry (UHPLC-QTOF-MS/MS) has been increasingly employed for rapid analysis of chemical profile or metabolites in TCMs [17]. Yet, one-dimensional liquid chromatography (1DLC) is unsatisfactory in the separation power of complex samples especially in TCMs. Compared with 1DLC, twodimensional liquid chromatography (2DLC) largely improved its peak capacity, thus obtaining higher resolution and separation capability, which have been considered as a better solution for the comprehensive characterization of TCMs $[16,18$, 19]. Furthermore, an enormous and specific natural product library used for $\mathrm{MS}^{1}$ matching could contribute to the rapid and precise assignment of the profiled compounds to a large extent [19]. Since most of the ingredients in TCMs are derived from secondary metabolites in plants, the same types of metabolites possess basic biosynthetic pathways, and their structures are typically correlated, which could be characterized with MS information by the same or similar fragmentation mode [20-22]. Accordingly, metabolite prediction based on structural correlations of the same types of compounds, known as MS-network, could enhance efficiency in the characterization of chemical components in TCM formulas [21]. Besides, the ultra-high-performance liquid chromatography coupled with triple-quadrupole tandem mass spectrometry (UHPLC-TQ-MS) provides a high sensitivity due to the multiple reaction monitoring (MRM) detection mode, which has also been demonstrated to be a powerful tool for the analysis of natural products [23].

In this study, an in-house chemical library was initially built on the basis of reported phytochemicals of the SJZD and its four herbs, which mainly contained saponins, flavonoids, and terpenoids. The structural correlations of these compounds were then analyzed, which led to the construction of three MS-networks of flavonoids, terpenoids, and saponins based on their basic skeletons. Meanwhile, an efficient offline reversed-phase $\times$ reversed-phase two-dimensional liquid chromatography quadrupole time-of-flight mass spectrometry $($ RPLC $\times$ RPLC-QTOF-MS) was developed to characterize the NPS components in SJZD. Furthermore, the major representative constituents were chosen for quantification with a rapid, sensitive, and validated method by UHPLCQTRAP ${ }^{\circledR}$-MS/MS. The aim of this study was to further uncover the bioactive material basis and lay a foundation for subsequent pharmacokinetics and action mechanism study.

\section{Materials and methods}

\section{Materials, reagents, and chemicals}

Liquiritin apioside, liquiritin, neoliquiritin, isoliquiritin apioside, isoliquiritin, ononin, naringin, liquiritigenin, poricoic acid A, poricoic acid B, poricoic acid C, glycyrrhetinic acid, dehydropachymic acid, pachymic acid, ginsenoside $\mathrm{Re}$, ginsenoside $\mathrm{Rg} 1$, ginsenoside Rf, isoliquiritigenin, ginsenoside $\mathrm{Rb} 1$, ginsenoside $\mathrm{Rg} 2$, ginsenoside $\mathrm{Rg} 3$, ginsenoside $\mathrm{Rg} 5$, 20(S)-ginsenoside $\mathrm{Rh} 1$, notoginsenoside R1, ginsenoside Ro, ginsenoside Rc, formononetin, ginsenoside Rb2, glycyrrhizic acid, ginsenoside Rd, ginsenoside Rk1, and atractylenolide III were purchased from Chengdu DeSiTe Biological Technology Co., Ltd. (Chengdu, China) or Sichuan Weikeqi Biological Technology Co., Ltd. (Chengdu, China). HPLC-grade acetonitrile was acquired from J\&K Scientific (China). LCMS-grade acetonitrile was acquired from Merck (Darmstadt, Germany). Deionized water was prepared from distilled water using a Milli-Q water purification system (Millipore, Bedford, MA, USA). All other reagents and chemicals were of analytical grade.

\section{Sample and reference standard preparation for qualitative and quantitative analyses}

SJZD sample was prepared according to our previous reports $[8,11]$. In brief, four individual dried herbs were mixed and boiled twice in distilled water. The solution was centrifuged, 
and then concentrated to $1 \mathrm{~g} / \mathrm{mL}$ (calculated as crude drug), resulting in the SJZD sample. Then, $8 \mathrm{~mL}$ of ethanol was added to $2 \mathrm{~mL}$ of SJZD sample with subsequent stirring, after which it was kept standing for $24 \mathrm{~h}$ to remove polysaccharides. The supernatant was centrifuged and then dried under vacuum at $60^{\circ} \mathrm{C}$. The residue was dissolved in $10 \mathrm{~mL}$ methanol-water $(50: 50, \mathrm{v} / \mathrm{v})$ and centrifuged at $14,000 \mathrm{rpm}$ for $10 \mathrm{~min}$ to obtain sample A. In total, $50 \mu \mathrm{L}$ of sample $\mathrm{A}$ was transferred into a $50-\mathrm{mL}$ volumetric flask with methanol-water $(50: 50, \mathrm{v} / \mathrm{v})$ to obtain sample B1. Similarly, $1 \mathrm{~mL}$ sample B1 was diluted ten times to acquire sample B2 in a $10-\mathrm{mL}$ volumetric flask.

Each standard was precisely weighed following standard procedures and dissolved in methanol-water (50:50-80:80, $\mathrm{v} / \mathrm{v})$. Standard solutions of liquiritin apioside, liquiritin, isoliquiritin apioside, isoliquiritin, ononin, liquiritigenin, ginsenoside Re, ginsenoside Rg1, ginsenoside Rf, isoliquiritigenin, ginsenoside $\mathrm{Rb} 1$, ginsenoside $\mathrm{Rg} 2$, ginsenoside Rc, formononetin, ginsenoside Rb2, glycyrrhizic acid, ginsenoside $\mathrm{Rd}$, ginsenoside $\mathrm{Rg} 3$, and ginsenoside $\mathrm{Rk} 1$ were prepared individually in the concentration ranging from 0.184 to $2.130 \mathrm{mg} / \mathrm{mL}$ and stored at $4{ }^{\circ} \mathrm{C}$ until use. A mixed working solution was prepared by diverting specific amounts of the 19 reference standard solutions into a $5-\mathrm{mL}$ volumetric flask. A set of standard solutions were prepared by appropriate dilution of the mixed stock solution with $50 \%$ methanol to obtain the calibration curve. All solutions were filtered through a $0.22-\mu \mathrm{m}$ membrane before analysis.

\section{Offline 2DLC-QTOF-MS/MS system}

The offline 2D separation was performed by using an Agilent 1260/1290 system, consisting of two binary pumps (1D: G7161B; 2D: G4220A), two diode array detectors (1D: G4212A; 2D: G4212B), a degasser (G1322A), a thermostatic column compartment (G1316C), and an autosample injector (G4226A) (Agilent Technologies, Inc., Santa Clara, CA, USA). The first-dimensional $\left({ }^{1} \mathrm{D}\right)$ separation was carried out on a 1260 system, equipped with an Agilent Eclipse XDB$\mathrm{C} 18(9.4 \mathrm{~mm} \times 250 \mathrm{~mm}, 5 \mu \mathrm{m})$. The mobile phase consisted of water (A) and methanol (B). The flow rate was set at $3 \mathrm{~mL} /$ $\mathrm{min}$. The gradient profile was as follows: $0-7 \mathrm{~min}, 10-18 \% \mathrm{~B}$; 7-26 min, 18-45\% B; 26-40 min, 45-95\% B; 40-45 min, 95$100 \% \mathrm{~B} ; 45-50 \mathrm{~min}, 100-10 \% \mathrm{~B}$. The column temperature was $25^{\circ} \mathrm{C}$; the injection volume was $100 \mu \mathrm{L}$ (sample A), and the detection wavelength was set at 210 and $254 \mathrm{~nm}$. The fraction was collected based on peak-profile and eluent from 0 to 4.89 min was labeled as Fr.1; from 4.90 to 14.06 was labeled as Fr.2; from 14.07 to 16.97 was labeled as Fr.3; from 16.98 to 26.00 was labeled as Fr.4; and from 26.01 to 46.00 was labeled as Fr.5. The replicate injection was repeated 10 times as in Fig. S1 (see Supplementary Information (ESM)), and the five fractions were concentrated in a vacuum and then evaporated to dryness by stead nitrogen flow at ambient temperature. Each residue was dissolved in $1 \mathrm{~mL} 50 \%$ methanol and then centrifuged at $14,000 \mathrm{rpm}$ for $10 \mathrm{~min}$ to obtain supernatant for ${ }^{2} \mathrm{D}$ analysis, respectively. A ZORBAX Eclipse Plus $\mathrm{C} 18(2.1 \mathrm{~mm} \times 100 \mathrm{~mm}, 1.8 \mu \mathrm{m})$ was used as ${ }^{2} \mathrm{D}$ column and eluted by a binary mobile phase consisting of water containing $0.1 \%$ (A) and acetonitrile (B) with a gradient as follows: 0-3 min, 5-9\% B; 3-20 min, 9-23\% B; 20-34 min, 23$32 \%$ B; 34-47 min, 32-37\% B; 47-54 min, 37-48\% B; 54$59 \mathrm{~min}, 48-59 \% \mathrm{~B} ; 59-60 \mathrm{~min}, 59-77 \% \mathrm{~B}$; 60-62 min, 7795\% B; 62-64 min, 95-5\% B; flow rate, $0.4 \mathrm{~mL} / \mathrm{min}$; column temperature, $40{ }^{\circ} \mathrm{C}$; injection volume, $2 \mu \mathrm{L}$.

An Agilent 6545 QTOF spectrometer equipped with a Dual AJS ESI source was operated in both negative and positive ion modes. The instrument was tuned and calibrated as recommended by the manufacturer. The optimized parameters were as follows: gas temperature, $320^{\circ} \mathrm{C}$; gas flow, $8 \mathrm{~L} / \mathrm{min}$; nebulizer gas pressure, $35 \mathrm{psi}$; sheath gas temperature, $350{ }^{\circ} \mathrm{C}$; sheath gas flow, $11 \mathrm{~L} / \mathrm{min}$; acquisition rates, 2 spectra/s for MS and 8 spectra/s for MS/MS; capillary potential, $3500 \mathrm{~V}$; fragmentor potential, $165 \mathrm{~V}$; collision energies, 5.00, 15.00, 30.00 , and $55.00 \mathrm{eV}$. The mass ranges of MS and MS/MS were from $\mathrm{m} / \mathrm{z}, 100$ to 1500 and $\mathrm{m} / \mathrm{z} 100$ to 1500 , severally.

\section{Data processing}

Data acquisition and processing were performed by the MassHunter software (B.08.00) in "preferred auto MS/MS" mode. The initial chemical library of four crude drugs of SJZD was built based on multiple available databases (e.g., Web of Science, SciFinder, CNKI, and TCMSP). The correlations in structures of each type of certain known compounds were summarized by the different substituent groups derived in several skeletons and were employed to predict potential metabolites by the partial transformation of skeleton structures such as hydrogenation $\left(\mathrm{H}_{2}\right)$, hydroxylation $(\mathrm{O})$, methylation $\left(\mathrm{CH}_{2}\right)$, acetylation $\left(\mathrm{C}_{2} \mathrm{H}_{2} \mathrm{O}\right)$, and glycosylation $\left(\mathrm{C}_{6} \mathrm{H}_{10} \mathrm{O}_{5}\right.$, $\mathrm{C}_{6} \mathrm{H}_{8} \mathrm{O}_{6}, \mathrm{C}_{5} \mathrm{H}_{8} \mathrm{O}_{4}$, and $\mathrm{C}_{6} \mathrm{H}_{10} \mathrm{O}_{4}$ ), which led to the construction of MS-network used for qualitative analysis. The MSnetwork analysis used for SJZD characterization is illustrated in Figs. 1 and 2, which produced more theoretical molecular formulas therewith [21]. The known and newly generated formulas were then incorporated into MassHunter Qualitative Analysis software (B.08.00). Preliminary annotation of the MS data was performed by searching the incorporated inhouse chemical library in a mode called "Find by Formula." Multiple experimental molecular weights were matched with the library data at once by an iterate searching algorithm, and the error range of retention time and mass was set at $0.4 \mathrm{~min}$ and $10 \mathrm{ppm}$. The structures of these matched molecular formulas were further validated using their tandem MS information. Some databases such as HMDB (https://hmdb.ca/) [24] and MassBank (http://www.massbank.jp/Index) [25] were also involved in the data processing. 


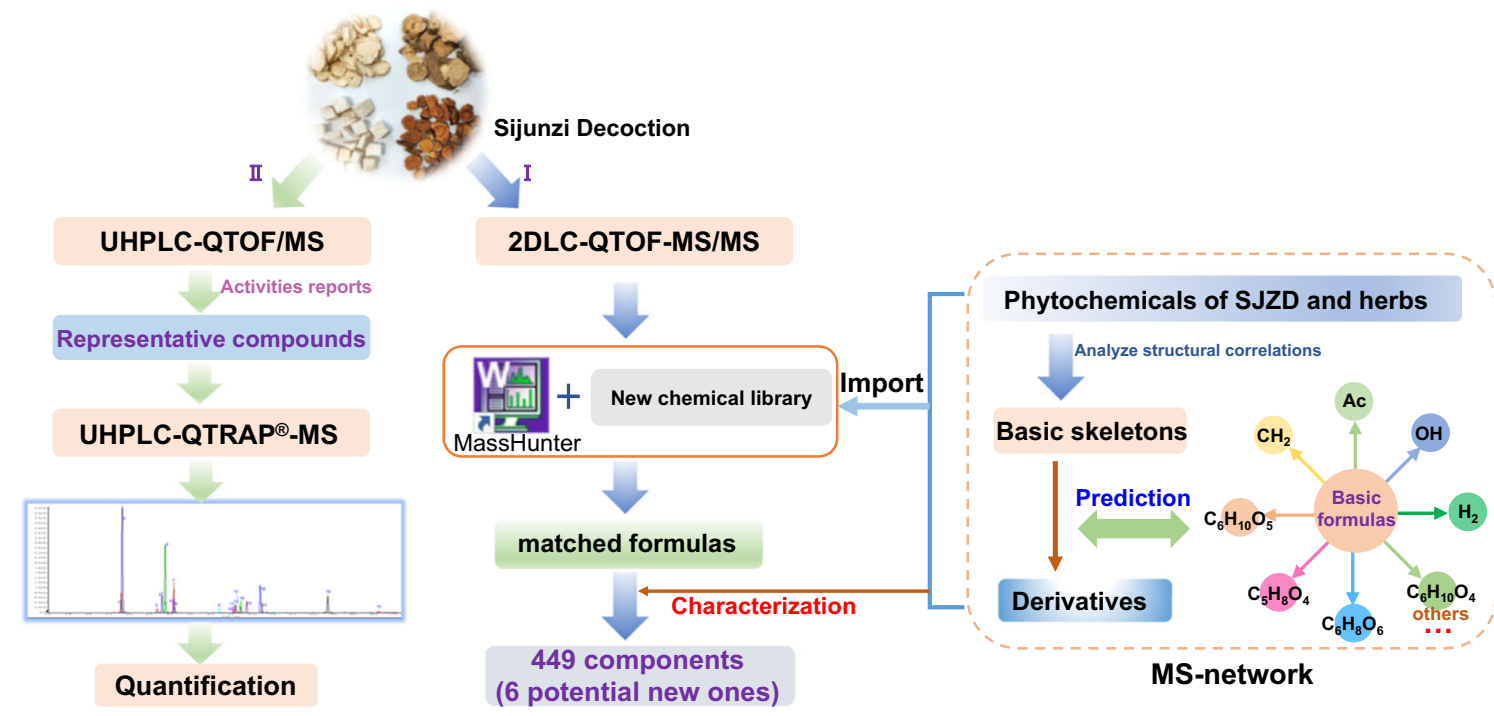

Fig. 1 A schematic diagram exhibiting the integrated strategy used for the comprehensive profiling and quantification of non-polysaccharide composition from SJZD

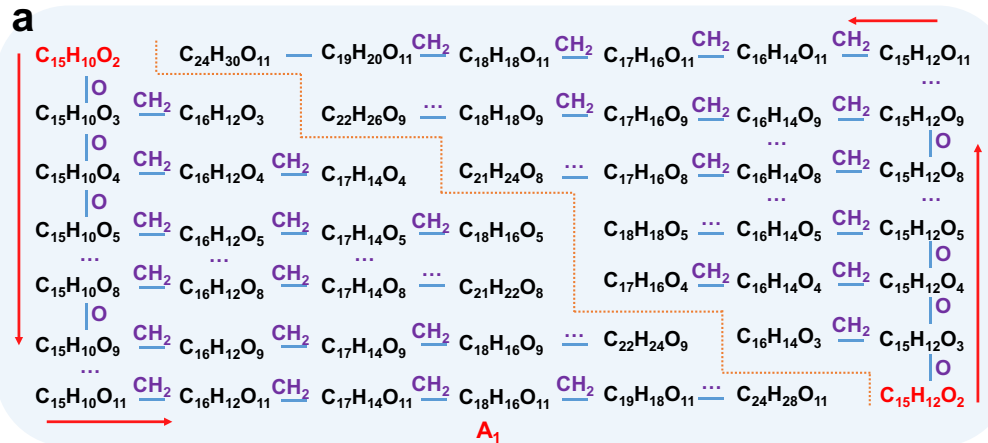

$\begin{array}{ccc}A_{1}+\text { Ac } & A_{2}+\text { Ac } & A_{n-1}+A c \\ A_{1}+\text { Glc } & A_{2}+\text { Glc } & A_{n-1}+\text { Glc } \\ A_{1}+\text { GluA } & A_{2}+\text { GluA } & A_{n-1}+\text { GluA } \\ A_{1}+\text { Rha } & A_{2}+\text { Rha } & A_{n-1}+\text { Rha } \\ A_{1}+\text { Apio } & A_{2}+\text { Apio } & A_{n-1}+\text { Apio } \\ A_{1}+\text { Xyl } & A_{2}+\text { Xyl } & A_{n-1}+\text { Xyl } \\ A_{1}+\text { Ara } & A_{2}+\text { Ara } & A_{n-1}+\text { Ara } \\ A_{2} & A_{3} & A_{n}\end{array}$

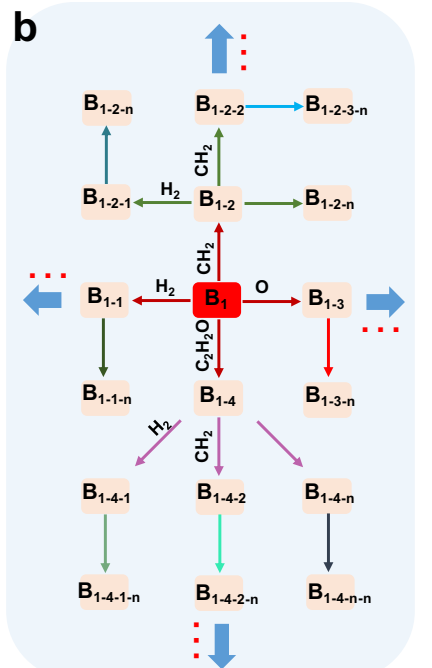

Fig. 2 MS-networks of flavonoids (a), terpenoids (b), and saponins (c) for SJZD. (a) The formulas in $\mathrm{A}_{1}$ were produced by adding $\mathrm{O}$ or $\mathrm{CH}_{2}$ to the two basic formulas $\left(\mathrm{C}_{15} \mathrm{H}_{10} \mathrm{O}_{2}\right.$ and $\left.\mathrm{C}_{15} \mathrm{H}_{12} \mathrm{O}_{2}\right) \cdot \mathrm{A}_{2}=\mathrm{A}_{1}+\mathrm{Ac}\left(\mathrm{C}_{2} \mathrm{H}_{2} \mathrm{O}\right)$; $\mathrm{A}_{1}+\mathrm{Glc}\left(\mathrm{C}_{6} \mathrm{H}_{10} \mathrm{O}_{5}\right) ; \mathrm{A}_{1}+$ GluA $\left(\mathrm{C}_{6} \mathrm{H}_{8} \mathrm{O}_{6}\right) ; \mathrm{A}_{1}+\mathrm{Rha}\left(\mathrm{C}_{6} \mathrm{H}_{10} \mathrm{O}_{4}\right) ; \mathrm{A}_{1}+$ $\mathrm{C}_{5} \mathrm{H}_{8} \mathrm{O}_{4}$ (Apio, Xyl, and Ara). Through the same strategy as for $\mathrm{A}_{2}, \mathrm{~A}_{3}$ to $A_{n}$ were built. (b) The MS-network of terpenoids was built based on $B_{1}$
C
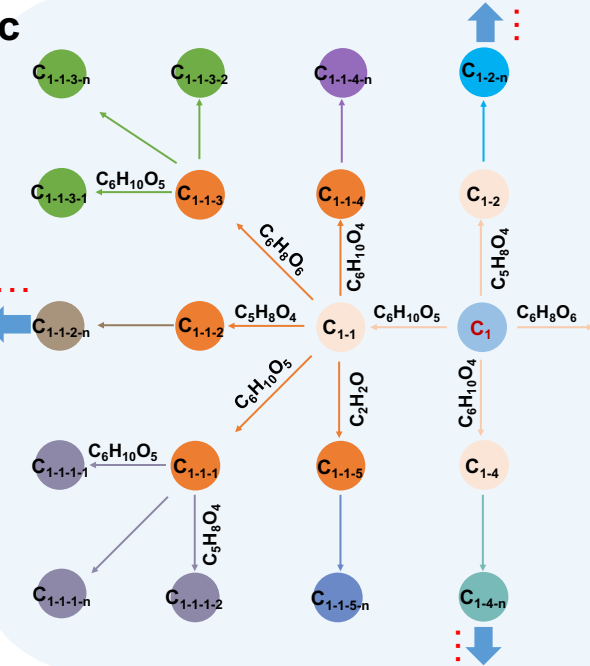

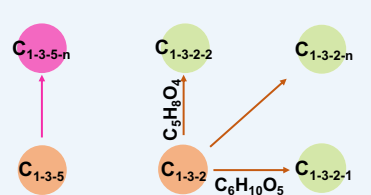

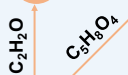

$\mathrm{C}_{1-3 \cdot 2} \underset{\mathrm{C}_{6} \mathrm{H}_{10} \mathrm{O}_{5}}{\longrightarrow} \mathrm{C}_{1-3-2 \cdot-1}$

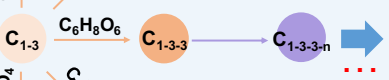

(several basic skeletons with their molecular formulas) with correlations $\left(\mathrm{H}_{2}, \mathrm{CH}_{2}, \mathrm{O}\right.$, and $\left.\mathrm{C}_{2} \mathrm{H}_{2} \mathrm{O}\right)$. (c) The MS-network of saponins was built based on $\mathrm{C}_{1}\left(\mathrm{C}_{30} \mathrm{H}_{52} \mathrm{O}_{3}, \mathrm{C}_{30} \mathrm{H}_{52} \mathrm{O}_{4}, \mathrm{C}_{30} \mathrm{H}_{48} \mathrm{O}_{3}, \mathrm{C}_{30} \mathrm{H}_{52} \mathrm{O}_{5}\right.$, and $\left.\mathrm{C}_{30} \mathrm{H}_{46} \mathrm{O}_{4}\right)$ with different substituent groups $\left(\mathrm{C}_{6} \mathrm{H}_{10} \mathrm{O}_{5}, \mathrm{C}_{6} \mathrm{H}_{8} \mathrm{O}_{6}\right.$, $\mathrm{C}_{6} \mathrm{H}_{10} \mathrm{O}_{4}, \mathrm{C}_{5} \mathrm{H}_{8} \mathrm{O}_{4}$, and $\mathrm{C}_{2} \mathrm{H}_{2} \mathrm{O}$ ) 


\section{UHPLC-QTRAP ${ }^{\oplus}$-MS/MS analysis conditions}

An ACQUITY UPLC system (Waters, Milford, MA, USA) coupled to a hybrid triple-quadrupole linear ion trap mass spectrometer (5500 QTRAP ${ }^{\circledR}$ ) with a turbo ion spray source from AB SCIEX (Foster City, CA, USA) was employed in this study. Equipment control and data analysis were performed using Analyst software ver. 1.5.1 (AB Sciex). UHPLC was equipped with a binary solvent delivery system and an autosample injector. Chromatographic separation was carried out at $45{ }^{\circ} \mathrm{C}$, using a ZORBAX Eclipse Plus C18 $(2.1 \mathrm{~mm} \times 100 \mathrm{~mm}, 1.8 \mu \mathrm{m})$ with mobile phases A $(0.1 \%$ formic acid in water) and $\mathrm{B}$ (acetonitrile). The flow rate was set at $0.4 \mathrm{~mL} / \mathrm{min}$; the gradient was operated as follows: 0 $18 \mathrm{~min}, 10-64 \% \mathrm{~B}$; the injection volume was $3 \mu \mathrm{L}$. Two cycles of weak-polarity (100\% acetonitrile) and highpolarity (10\% acetonitrile) solvent washing of the injecting system were carried out between injections.

The main parameters of QTRAP ${ }^{\circledR}$-MS/MS performed in negative ionization mode were set as follows: ion spray voltage, $-4500 \mathrm{~V}$; probe temperature, $650{ }^{\circ} \mathrm{C}$; curtain gas, $45 \mathrm{psi}$; nebulizer gas (GS1), 40 psi; auxiliary gas (GS2), 45 psi; collision-activated dissociation, medium. Nitrogen was employed as a nebulizer and collision gas. The MRM was employed in the acquisition mode, which selected ions at the first $(Q 1)$ and third quadrupole $(Q 3)$. For the appropriate fragmentation patterns of $\mathrm{m} / \mathrm{z}(Q 1)$ and $\mathrm{m} / \mathrm{z}(Q 3)$ for analytes in MRM mode, each single standard solution was further optimized and listed in Table S1 with selected quantifier and qualifier ions (see ESM). Due to high content in SJZD and high response in MS of liquiritin and liquiritin apioside, the four compounds liquiritin, isoliguiritin, liquiritin apioside, and isoliquiritin apioside were analyzed in sample B2, while the remaining were analyzed in sample B1 to reduce the carryover effect in the continuous injections.

\section{UPLC-MS/MS method validation}

According to the European Medicines Agency (EMEA) guidelines [26], the developed quantitative method was evaluated in terms of linearity, precision, repeatability, stability, and accuracy. A series of mixed standard solutions were prepared through precise stepwise dilution. Several standard solutions of the 19 analytes were analyzed, and then the calibration curve for each analyte was established using linear regression stimulation of the peak area $(y)$ versus concentration $(x)$ to determine the correlation coefficient $\left(R^{2}\right)$. The precision of the method was evaluated by analyzing the 19 standard compounds. The same mixed standard solution was tested for six replicates within 1 day to get the intra-day precisions, and examined in duplicates for consecutive 3 days to obtain inter-day precisions. For the repeatability test, six SJZD samples were extracted and processed to obtain 6 sample B1 and 6 sample B2 solutions, respectively, in line with the methods mentioned above. The obtained sample B1 and sample B2 solutions were analyzed in parallel, and RSD calculated the variations to evaluate the repeatability of this method. The sample B1 and B2 solutions were stored in a sample manager at $8{ }^{\circ} \mathrm{C}$ and analyzed at $0,6,12$, and $24 \mathrm{~h}$, respectively, to investigate the stability. The spike recovery rate is employed to further evaluate the accuracy of this method. The SJZD sample was spiked with mixed standard with low, medium, and high concentrations (75\%/100\%/125\%), followed by extraction (ethanol precipitation), processing, and quantification in accordance with the methods mentioned above in three parallel operations. The results from the determination of recovery were expressed as the percentage of the analytes recovered by the assay.

\section{Results}

\section{The construction and optimization of offline 2DLC- QTOF-MS/MS}

The constituents of reported SJZD mainly included flavonoids, saponins, and terpenoids, which could be well separated by RPLC. Compared to 1DLC, 2DLC has been proven to have greater advantages in separating complex components in TCM. Among them, RPLC $\times$ RPLC is widely used in the separation of saponins, flavonoids, and phenolic compounds, which suggests good resolution capabilities [27-31]. Hence, an offline RP $\times$ RP 2DLCMS system was developed to analyze the NPSs of SJZD with optimized LC and MS conditions.

The first-dimensional separation In an offline 2DLC system, ${ }^{1}$ DLC performs the preliminary separation of natural products. Based on the distribution of chromatographic peaks, the elution compounds could be collected in different fractions, resulting in better separation in ${ }^{2}$ DLC and enrichment of some minor or trace constituents. In order to realize the quick enrichment of fractions for ${ }^{2}$ DLC detection, Eclipse XDB-C18 with high loading capacity at once was selected. Since different elution solvents of ${ }^{1} \mathrm{D}$ and ${ }^{2} \mathrm{D}$ could improve the resolution and the use of acetonitrile instead of methanol could lead to the remarkable retention time shift, methanol/water was used as the ${ }^{1} \mathrm{D}$ mobile phase, and acetonitrile/acidified water $(0.1 \%$ formic acid) was used as the ${ }^{2} \mathrm{D}$ mobile phase therewith $[28$, 29]. Peak-dependent fraction collection was adopted to reduce the complexity of the 2DLC-MS detection, thus resulting in the five fractions as shown in Fig. S1 (see ESM).

Optimization of the second-dimensional RPLC and MS conditions The selection of the column for ${ }^{2} \mathrm{D}$ separation was based on the column efficiency and resolution of major 
components. Four columns, namely ACQUITY BEH C18 $(2.1 \times 100 \mathrm{~mm}, 1.7 \mu \mathrm{m})$, ZORBAX Eclipse Plus C18 $(2.1 \mathrm{~mm} \times 100 \mathrm{~mm}, 1.8 \mu \mathrm{m})$, Agilent Poroshell $120 \mathrm{EC}-$ C18 $(3.0 \mathrm{~mm} \times 150 \mathrm{~mm}, 2.7 \mu \mathrm{m})$, and ZORBAX SB-C8 $(3.0 \mathrm{~mm} \times 100 \mathrm{~mm}, 1.8 \mu \mathrm{m}$ ), were evaluated (see ESM Fig. S2). As a result, ZORBAX Eclipse Plus C18 showed better performance than others. UHPLC conditions were also optimized, including mobile phase, flow rate, and column temperature, to obtain a good separation. Finally, acetonitrile/acidified water $(0.1 \%$ formic acid), which showed good peak shapes, more amounts of peaks, and better intensity, was selected as the mobile phase. The flow rate and temperature were $0.4 \mathrm{~mL} / \mathrm{min}$ and $40{ }^{\circ} \mathrm{C}$, respectively. For the MS conditions, both the positive and negative modes were analyzed. Still, the intensity of NPSs in negative mode is higher than that in positive mode. Besides, more compounds can be detected in negative mode according to the base peak intensity chromatograms (BPCs), as shown in Fig. S3 (see ESM). Therefore, the negative detection was chosen for identification. Other MS parameters have also been analyzed and optimized, such as capillary voltage, atomizing gas pressure, and drying gas flow rate. The results indicated that this study's newly developed qualitative method was appropriate for detecting its chemical constituents.

\section{Characterization of the NPSs in SJZD}

In the current study, sufficient abundance ions and structural information were obtained through negative mode. In total, four hundred forty-nine compounds were identified or tentatively characterized, including 100 flavonoids, 38 terpenoids, 276 saponins, and 35 other compounds. Corresponding chromatographic and mass spectral data of these compounds are shown in Tables S2-5 (see ESM2). Thirty-two compounds were further confirmed by comparison with reference standards, and 6 potentially novel ones were deduced from known structures by MS-network interpretation. The molecular formulas of all compounds matched their quasi-molecular ions such as $[\mathrm{M}-\mathrm{H}]^{-},[\mathrm{M}+\mathrm{FA}-\mathrm{H}]^{-}$within the mass error of $10 \mathrm{ppm}$. Due to the high-resolution capability of 2DLC-MS, more compounds, including isomers, could be characterized. Also, when a matched formula could be assigned to several isomers, the structure reported from the four individual herbs of SJZD before had a higher possibility than the others. Eventually, the structures of isomers were deduced by MS-network, fragment ions, and some online databases, such as ChemSpider (www.chemspider.com) [32], HMDB (https://hmdb.ca/) [24], and MassBank (http:// www.massbank.jp) [25].

\section{Flavonoids}

The basic framework of flavonoids is " $\mathrm{C}_{6}-\mathrm{C}_{3}-\mathrm{C}_{6}$." The different substituent groups are often composed of hydroxyl, methoxy, acetyl, and glycosyl. Therefore, the MS-network of flavonoids was built based on two basic flavonoidsflavone $\left(\mathrm{C}_{15} \mathrm{H}_{10} \mathrm{O}_{2}\right)$ and flavanone $\left(\mathrm{C}_{15} \mathrm{H}_{12} \mathrm{O}_{2}\right)$ - which is shown in Fig. 2a. Herein, more formulas could be matched in MS software. Once one formula was matched, it would be traced in MS-network and initial chemical library firstly, and further validated by its MS/MS information, which led to the identification of 100 flavonoids, including 2 potential novel ones. These structures could be classed as flavonoid aglycones and flavonoid glycosides.

Flavonoid aglycones Retro Diels-Alder (RDA) fragmentation mechanism was common for flavonoids in MS. The neutral losses of $18.0106 \mathrm{Da}\left(\mathrm{H}_{2} \mathrm{O}\right), 15.0235 \mathrm{Da}\left(\mathrm{CH}_{3}\right), 14.0157 \mathrm{Da}$ $\left(\mathrm{CH}_{2}\right), 27.9949 \mathrm{Da}(\mathrm{CO})$, and $43.9898 \mathrm{Da}\left(\mathrm{CO}_{2}\right)$ were also the characteristic fragmentation behaviors for flavone aglycones. For example, peak A78 showed a parent $[\mathrm{M}-\mathrm{H}]^{-}$ion at $\mathrm{m} / \mathrm{z}$ 299.0564, indicating a formula of $\mathrm{C}_{16} \mathrm{H}_{12} \mathrm{O}_{6}$. Diagnostic ions at $m / z 284.0306\left[\mathrm{M}-\mathrm{H}-\mathrm{CH}_{3}\right]^{-}, m / z 165.0188\left[\mathrm{C}_{8} \mathrm{H}_{5} \mathrm{O}_{4}\right]^{-}$, and $\mathrm{m} / \mathrm{z} 151.0029\left[\mathrm{C}_{7} \mathrm{H}_{3} \mathrm{O}_{4}\right]^{-}$were suggestive of a flavonoid skeleton with a hydroxyl and one methoxy in ring $\mathrm{A}$, as shown in Figs. 3 and 4a. B-ring bearing two hydroxyls was then deduced via the molecular formula and the reference [33], suggesting the identification of 7-O-methylluteolin for A78. The daughter ions at $\mathrm{m} / \mathrm{z} 271.0606, \mathrm{~m} / \mathrm{z} 256.0369$, and $\mathrm{m} / \mathrm{z}$ 240.0423 were indicative of losses of $\mathrm{CO}, \mathrm{C}_{2} \mathrm{H}_{3} \mathrm{O}$, and $\mathrm{CH}_{3} \mathrm{O}_{2}$, respectively, which validated the speculation. A50 acquired a formula of $\mathrm{C}_{15} \mathrm{H}_{12} \mathrm{O}_{4}$ as the mother ion at $\mathrm{m} / \mathrm{z}$ $255.0662[\mathrm{M}-\mathrm{H}]^{-}$, which yielded two characteristic daughter ions of flavanones at $\mathrm{m} / \mathrm{z} 135.0085\left[\mathrm{C}_{7} \mathrm{H}_{3} \mathrm{O}_{3}\right]^{-}$and $\mathrm{m} / \mathrm{z}$ $119.0497\left[\mathrm{C}_{8} \mathrm{H}_{8} \mathrm{O}\right]^{-}$. Consequently, it was identified as liquiritigenin by comparing elution time and MS fragment ions with reference standard. $\mathbf{A 7 5}$ revealed diagnostic ions at $m / z 267.0665[\mathrm{M}-\mathrm{H}]^{-}$and $m / z 252.0435\left[\mathrm{M}-\mathrm{H}-\mathrm{CH}_{3}\right]^{-}$, which was finally confirmed by formononetin standard. A81 were presumed as licoisoflavanone in view of the $[\mathrm{M}-\mathrm{H}]^{-}$ion at $\mathrm{m} / \mathrm{z}$ 353.1017 together with its daughter ion at $\mathrm{m} / \mathrm{z} 227.0719$ [M$\left.\mathrm{H}-\mathrm{C}_{6} \mathrm{H}_{6} \mathrm{O}_{3}\right]^{-}$and $m / z 125.0240\left[\mathrm{C}_{6} \mathrm{H}_{6} \mathrm{O}_{3}-\mathrm{H}\right]^{-}$, which conformed to the fragment ion reported in existing reference [34]. The loss of $\mathrm{C}_{4} \mathrm{H}_{8}$ was observed in $\mathbf{A 8 5}$ by the mother ion at $\mathrm{m} / \mathrm{z} 353.1028[\mathrm{M}-\mathrm{H}]^{-}$and its daughter ion at $\mathrm{m} / \mathrm{z} 297.0423$ $\left[\mathrm{M}-\mathrm{H}-\mathrm{C}_{4} \mathrm{H}_{8}\right]^{-}$, resulting in the characterization of glycyrrhisoflavone [34]. A87 was assigned a formula of $\mathrm{C}_{21} \mathrm{H}_{20} \mathrm{O}_{5}$ according to the quasi-molecular ion at $\mathrm{m} / \mathrm{z}$ 351.1238 [M-H] $]^{-}$. Diagnostic ions at $\mathrm{m} / z 336.0998$ and $\mathrm{m} / \mathrm{z}$ 281.0452 were deduced to be the losses of $\mathrm{CH}_{3}$ and $\mathrm{C}_{5} \mathrm{H}_{10}$, respectively, which implied the presence of the methoxy and isopentene group. It was tentatively identified as gancaonin A, which had been reported in reference [35]. A88 displayed a 


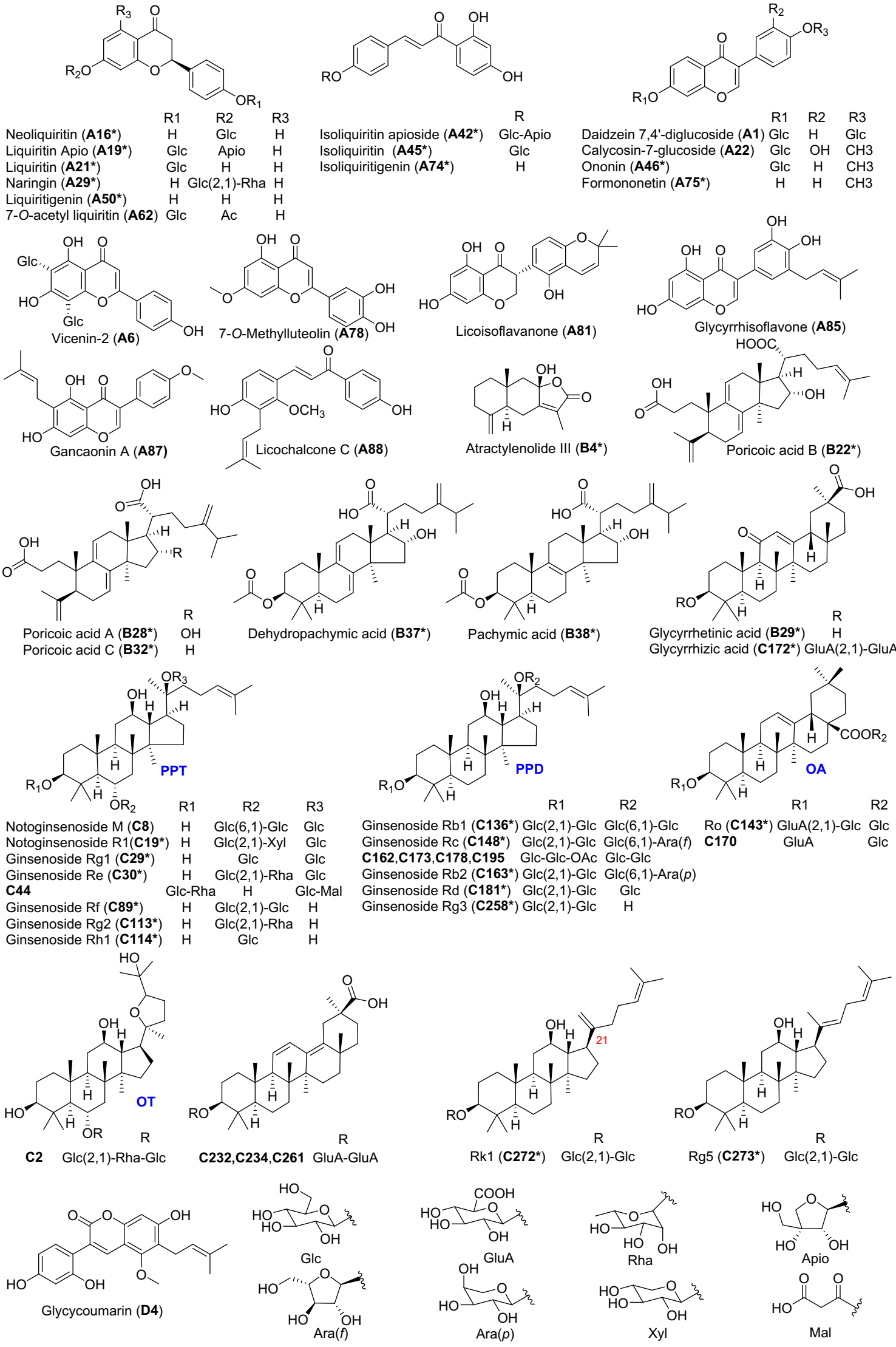

Fig. 3 Potential chemical structures of partial NPSs in SJZD. The flavonoids (A), terpenoids (B), saponins (C), and other components (D) were classified. The superscript $*$ means that the compound was identified by a standard substance 
Fig. 4 Potential fragment pathways of A78 (a), A22 (b), A6 (c), B4 (d), B37 (e), and D30 (f)

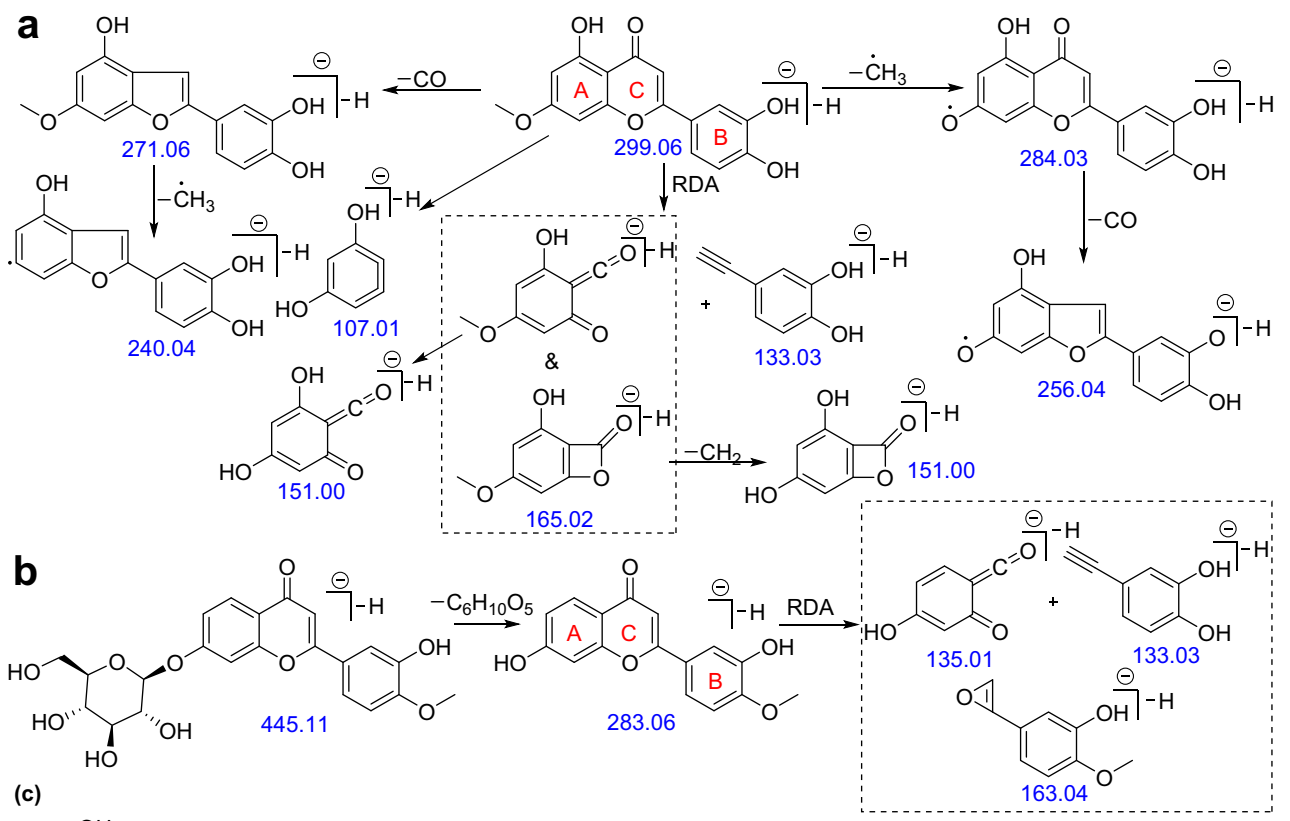

(c)

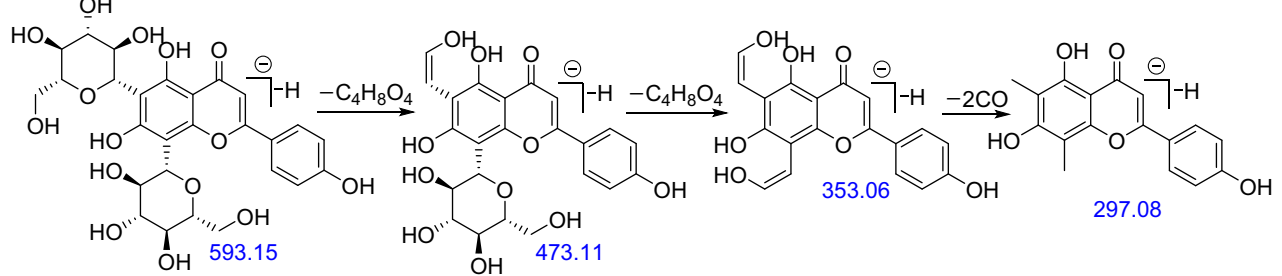

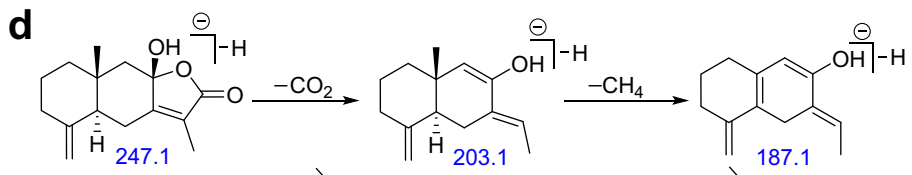

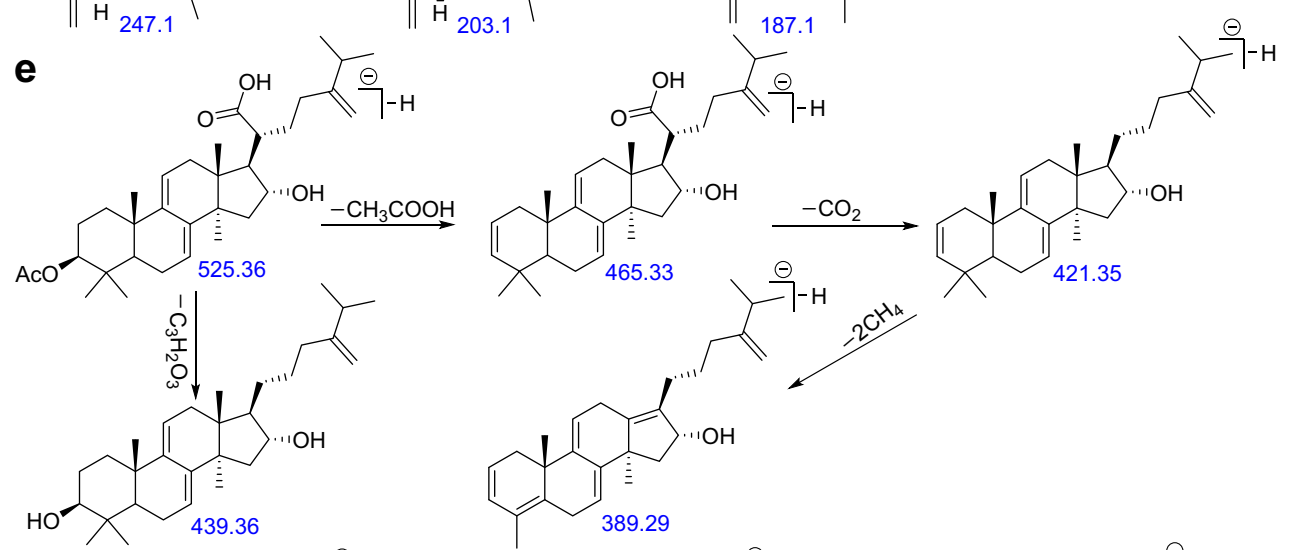

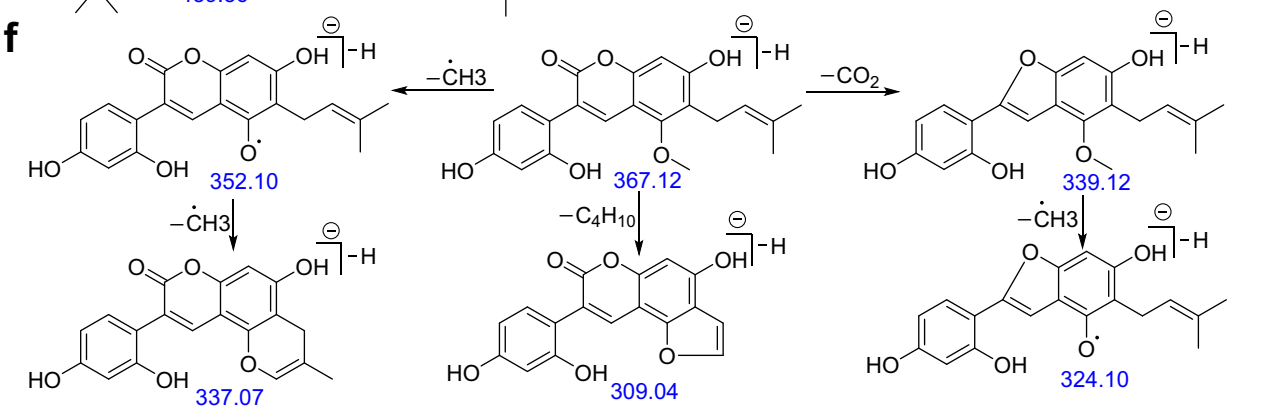


mother ion at $m / z 337.1443[\mathrm{M}-\mathrm{H}]^{-}$and typical fragment ions at $\mathrm{m} / \mathrm{z}, 305.1170\left[\mathrm{M}-\mathrm{H}-\mathrm{CH}_{3} \mathrm{OH}\right]^{-}, \mathrm{m} / z 243.1024$ [M-H$\left.\mathrm{C}_{6} \mathrm{H}_{6} \mathrm{O}\right]^{-}, \mathrm{m} / \mathrm{z}, 201.0918\left[\mathrm{M}-\mathrm{H}-\mathrm{C}_{8} \mathrm{H}_{8} \mathrm{O}_{2}\right]^{-}, \mathrm{m} / \mathrm{z} 187.0762$ $\left[\mathrm{M}-\mathrm{H}-\mathrm{C}_{8} \mathrm{H}_{8} \mathrm{O}_{2}-\mathrm{CH}_{2}\right]^{-}$, and $\mathrm{m} / z \quad 120.0215[\mathrm{M}-\mathrm{H}-$ $\left.\mathrm{C}_{14} \mathrm{H}_{17} \mathrm{O}_{2}\right]^{-}$, which were consistent with the product ions of licochalcone $\mathrm{C}$ [36].

Flavonoid glycosides These compounds can be classified as flavonoid $O$-glycoside and flavonoid $C$-glycoside. The elimination of saccharide groups regularly occurs in flavonoid $O$ glycoside, and the characteristic neutral losses of sugars [Glucose (Glc, 162.05 Da), Apiose (Apio, 132.04 Da), rhamnose (Rha, $146.06 \mathrm{Da})$, xylose/arabinose (Xyl/Ara, 132.04 Da), glucuronic acid (GluA, 176.03 Da)] could be observed in MS/MS spectrum. The process of qualitative analysis for some peaks was described so as to provide examples. Peak A21 showed a parent ion at $m / z 417.1189[\mathrm{M}-\mathrm{H}]^{-}$and a daughter ion at $m / z 255.0657\left[\mathrm{M}-\mathrm{H}-\mathrm{C}_{6} \mathrm{H}_{10} \mathrm{O}_{5}\right]^{-}$, indicating the presence of a glucosyl. Featured ions at $\mathrm{m} / \mathrm{z}, 135.0090$ and $\mathrm{m} / \mathrm{z}$ 119.0503 were produced due to the RDA fragmentation. It was further identified as liquiritin by comparing it with the standard. $\mathbf{A 6 2}$ generated $[\mathrm{M}-\mathrm{H}]^{-}$ion 42 Da greater than that of A21, suggesting that it might be acetyl derivative of liquiritin. The daughter ions at $m / z 297.0754\left[\mathrm{M}_{-} \mathrm{C}_{6} \mathrm{H}_{10} \mathrm{O}_{5}\right]^{-}$ and $m / z 255.0654\left[\mathrm{M}_{-} \mathrm{C}_{6} \mathrm{H}_{10} \mathrm{O}_{5}-\mathrm{C}_{2} \mathrm{H}_{2} \mathrm{O}\right]^{-}$supported the assumption, implying that $\mathbf{A 6 2}$ might be 7-O-acetyl liquiritin. Similarly, the fragment ion at $m / z 255.0665$ of $\mathbf{A 2 9}$ was also observed, suggesting it as naringin which was further verified by the reference compound. The quasi-molecular ion at $\mathrm{m} / \mathrm{z}$ $445.1142[\mathrm{M}-\mathrm{H}]^{-}$and its fragment ion at $m / z 283.0604[\mathrm{M}-\mathrm{H}-$ $\left.\mathrm{C}_{6} \mathrm{H}_{10} \mathrm{O}_{5}\right]^{-}$of $\mathbf{A 2 2}$ were suggestive of a glucosyl calycosin. The RDA fragment ions at $\mathrm{m} / \mathrm{z} 163.0396\left[\mathrm{C}_{9} \mathrm{H}_{7} \mathrm{O}_{3}\right]^{-}, \mathrm{m} / \mathrm{z}$ $135.0077\left[\mathrm{C}_{7} \mathrm{H}_{3} \mathrm{O}_{3}\right]^{-}$, and $m / z$ 133.0291 $\left[\mathrm{C}_{8} \mathrm{H}_{5} \mathrm{O}_{2}\right]^{-}$led to the identification of calycosin-7-glucoside for A22 (Fig. 4b). A1 with adduct [M+FA-H] $]^{-}$ion at $m / z 623.1636$ and $[\mathrm{M}-\mathrm{H}]^{-}$ ion at $\mathrm{m} / \mathrm{z}$ 577.1541, yielded diagnostic ions at $\mathrm{m} / \mathrm{z} 415.1030$ $\left[\mathrm{M}-\mathrm{H}-\mathrm{C}_{6} \mathrm{H}_{10} \mathrm{O}_{5}\right]^{-}, 253.0505\left[\mathrm{M}-\mathrm{H}-2 \mathrm{C}_{6} \mathrm{H}_{10} \mathrm{O}_{5}\right]^{-}$, suggesting successive losses of glucosyl. Compared with the fragment ions reported in reference, it was tentatively characterized as daidzein 7,4'-diglucoside [37]. Parent ion at $\mathrm{m} / \mathrm{z} 593.1509$ [M$\mathrm{H}]^{-}$of $\mathbf{A 6}$ was indicative of the $\mathrm{C}_{27} \mathrm{H}_{30} \mathrm{O}_{15}$ formula. The neutral losses of $\mathrm{C}_{4} \mathrm{H}_{8} \mathrm{O}_{4}(120 \mathrm{Da})$ and $\mathrm{C}_{3} \mathrm{H}_{6} \mathrm{O}_{3}(90 \mathrm{Da})$ were observed by the diagnostic ions at $[(\mathrm{m} / \mathrm{z}, 593.1504, \mathrm{~m} / \mathrm{z}$ 473.1078, $\mathrm{m} / \mathrm{z} 353.0665) ;(\mathrm{m} / \mathrm{z} 593.1504, \mathrm{~m} / \mathrm{z} 503.1186) ;(\mathrm{m} /$ $z$ 473.1078, $\mathrm{m} / \mathrm{z}$ 383.0762)], suggesting that $\mathbf{A 6}$ is a $C$ diglycoside flavonoid [38]. It was presumed as vicenin-2 due to the characteristic ions at $m / z 353.0665[\mathrm{M}-\mathrm{H}-$ $\left.\mathrm{C}_{8} \mathrm{H}_{16} \mathrm{O}_{8}\right]^{-}$and $m / z 297.0762$ [M-H- $\left.\mathrm{C}_{10} \mathrm{H}_{16} \mathrm{O}_{10}\right]^{-}$, as shown in Fig. 4c. Peak A25 provided fragment ions at $m / z 635.1621$ $[\mathrm{M}-\mathrm{H}]^{-}, m / z 591.1724\left[\mathrm{M}-\mathrm{H}-\mathrm{CO}_{2}\right]^{-}, m / z 549.1606[\mathrm{M}-\mathrm{H}-$ $\mathrm{CO}_{2}$-acetyl] $]^{-}, \mathrm{m} / \mathrm{z} 417.1185$ [M-H-acetyl-GluA] ${ }^{-}$, and $\mathrm{m} / \mathrm{z}$ 255.0666 [M-H-GluA-Glc-acetyl] $]^{-}$. The neutral losses of saccharide groups also occurred in peak $\mathbf{A 5 2}$ for the daughter ions at $m / z 433.1464$ [M-H-Apio] ${ }^{-}$and $m / z 271.0976$ [M-HApio-Glc] $]^{-}$. The aglycone might be vestitol due to the fragment ions at $\mathrm{m} / \mathrm{z} 271.0976, \mathrm{~m} / \mathrm{z} 255.0650$, and $\mathrm{m} / \mathrm{z}$ 135.0444 [35]. The structures of $\mathbf{A 2 5}$ and A52, which are depicted in Fig. S4A-B (see ESM), were inferred according to the structural correlations of flavonoids from the MS-network and may serve as novel structures not yet found in the SciFinder database.

\section{Terpenoids}

The main types of terpenoids reported in SJZD include triterpene carboxylic acids and sesquiterpenes, which mainly originate from Atractylodes Macrocephalae Rhizoma and Poria. The substituent groups often contain hydroxyl, methyl, methoxyl, and acetoxyl. Accordingly, the MS-network of terpenoids derived with $\mathrm{H}_{2}, \mathrm{CH}_{2}, \mathrm{O}$, and $\mathrm{C}_{2} \mathrm{H}_{2} \mathrm{O}$ was established from the several basic skeleton structures such as poricoic acid $\mathrm{C}\left(\mathrm{C}_{31} \mathrm{H}_{46} \mathrm{O}_{4}\right)$, dehydrotumulosic acid $\left(\mathrm{C}_{31} \mathrm{H}_{48} \mathrm{O}_{4}\right)$, dehydrotrametenoic acid $\left(\mathrm{C}_{30} \mathrm{H}_{46} \mathrm{O}_{3}\right)$, and atractylenolide II $\left(\mathrm{C}_{15} \mathrm{H}_{20} \mathrm{O}_{2}\right)[15,39]$. In addition, the terpenoids tend to eliminate small neutral fragments like $\mathrm{H}_{2} \mathrm{O}$, the methyl $\left(\mathrm{CH}_{2}, \mathrm{CH}_{3}\right.$. , and $\left.\mathrm{CH}_{4}\right), \mathrm{CO}_{2}, \mathrm{C}_{2} \mathrm{H}_{2} \mathrm{O}$, and $\mathrm{C}_{2} \mathrm{H}_{4} \mathrm{O}_{2}$ under collisioninduced decomposition conditions, which could also contribute to the quick assignment of these components. A total of 38 terpenoids were unambiguously or tentatively identified. Peak B4 was unequivocally identified as atractylenolide III via comparison with the reference standard. Its mother ion at $\mathrm{m} / \mathrm{z}, 247.1338[\mathrm{M}-\mathrm{H}]^{-}$and daughter ions at $\mathrm{m} / \mathrm{z}, 203.1448$ $\left[\mathrm{M}-\mathrm{H}-\mathrm{CO}_{2}\right]^{-}, m / z, 187.1098\left[\mathrm{M}-\mathrm{H}-\mathrm{C}_{2} \mathrm{H}_{4} \mathrm{O}_{2}\right]^{-}$were indicative of the possible fragmentation pathways, as depicted in Fig. 4d. Peak B37 revealed parent ion at $m / z 525.3578[\mathrm{M}-\mathrm{H}]^{-}$with a molecular formula $\mathrm{C}_{33} \mathrm{H}_{50} \mathrm{O}_{5}$. The diagnostic ions at $\mathrm{m} / \mathrm{z}$ $465.3346\left[\mathrm{M}-\mathrm{H}-\mathrm{CH}_{3} \mathrm{COOH}\right]^{-}, \mathrm{m} / z 421.3512[\mathrm{M}-\mathrm{H}-$ $\left.\mathrm{CH}_{3} \mathrm{COOH}-\mathrm{CO}_{2}\right]^{-}$, and $\mathrm{m} / z 389.2883$ [M-H-CH${ }_{3} \mathrm{COOH}-$ $\left.\mathrm{CO}_{2}-2 \mathrm{CH}_{4}\right]^{-}$were observed, which indicated the existence of an acetoxyl and a carboxyl group. B38 exhibited similar fragmentation pathways as previously reported triterpenoid [39], and was finally identified as dehydropachymic acid by the corresponding standard substance.

\section{Saponins}

Saponins occupy a large proportion in SJZD, which mainly came from Ginseng Radix and Glycyrrhizae Radix. These characterized saponins, based on the difference in sapogenin, could be classified into several subclasses. For example, the ginsenosides mainly contained the following: protopanaxadiol (PPD) type, protopanaxatriol (PPT) type, oleanolic acid (OA) type, and octillol (OT) type [19, 40], of which the primary fragmentation pattern was the successive cleavage at the glycosidic linkages. The typical product ions 

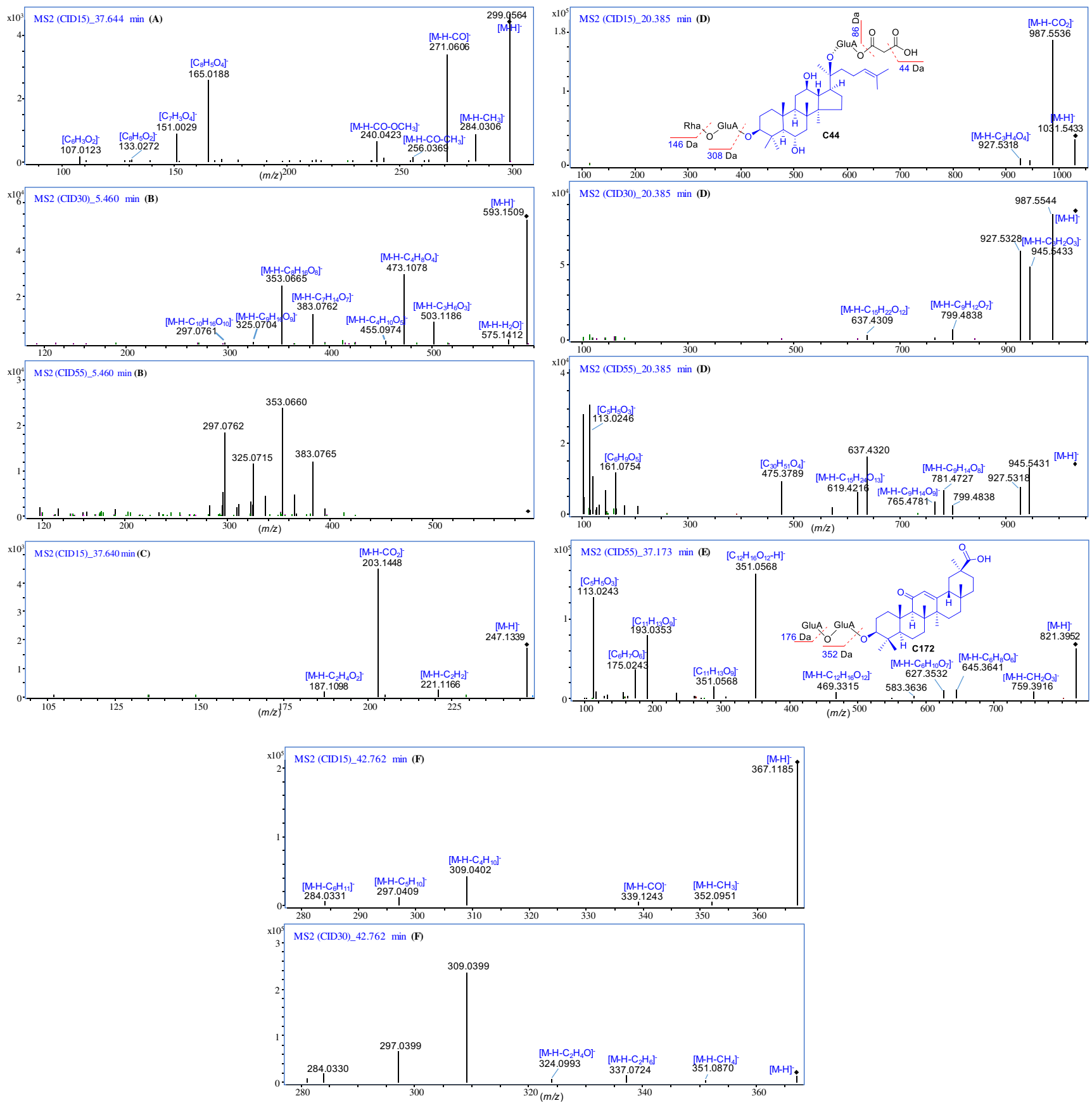

Fig. 5 Tandem mass spectra of A78 (A), A6 (B), B4 (C), C44 (D), C172 (E), and D30 (F)

could be assigned to the sapogenins $(\mathrm{m} / \mathrm{z} 475.38 / 391.29$ for PPT, 459.38/375.29 for PPD, 455.35 for OA, and 491.37/ 415.32 for OT) and glycosyl linkages $(\mathrm{m} / \mathrm{z}, 351.06 / 193.04$ for GluA-GluA; m/z 323.09 for Glc-Glc; $m / z$ 497.1159/ 321.0806 for GluA-GluA-Rha, and so on), which contribute to the quick identification of unknown compounds. Therefore, the MS-network (Fig. 2c) for saponins was built based on several basic sapogenins-PPD $\left(\mathrm{C}_{30} \mathrm{H}_{52} \mathrm{O}_{3}\right)$; PPT $\left(\mathrm{C}_{30} \mathrm{H}_{52} \mathrm{O}_{4}\right)$; OA $\left(\mathrm{C}_{30} \mathrm{H}_{48} \mathrm{O}_{3}\right)$; OT $\left(\mathrm{C}_{30} \mathrm{H}_{52} \mathrm{O}_{5}\right)$; glycyrrhetinic acid $\left(\mathrm{C}_{30} \mathrm{H}_{46} \mathrm{O}_{4}\right)$. Peaks of C6, C8, C14, C20, C23, and C79 were suggestive of the same formula $\mathrm{C}_{48} \mathrm{H}_{82} \mathrm{O}_{19}$ based on the quasi- molecular ions at $961.54[\mathrm{M}-\mathrm{H}]^{-}$or $1007.54[\mathrm{M}+\mathrm{FA}-\mathrm{H}]^{-}$, which all yielded the characteristic ions corresponding to PPT type at $m / z$ 475.38. The successive loss of Glc was deduced by the daughter ions at $m / z 799.48\left[\mathrm{M}-\mathrm{H}-\mathrm{C}_{6} \mathrm{H}_{10} \mathrm{O}_{5}\right]^{-}$, $m / z 637.4277\left[\mathrm{M}-\mathrm{H}-2 \mathrm{C}_{6} \mathrm{H}_{10} \mathrm{O}_{5}\right]^{-}$, and $m / z 475.38[\mathrm{M}-\mathrm{H}-$ $\left.3 \mathrm{C}_{6} \mathrm{H}_{10} \mathrm{O}_{5}\right]^{-}$, which indicated that these compounds might be notoginsenoside $\mathrm{M}$ and its isomers [20]. Peak $\mathbf{C 1 9}$ was also assigned as PPT-type ginsenoside as the typical ion at $m / z$ 475.38. The consecutive elimination of sugar residues including Xyl, Glc, and Glc was observed by the parent ion at $m / z, 931.53[\mathrm{M}-\mathrm{H}]^{-}$and its daughter ions at $m / z 799.48[\mathrm{M}-$ 
$\left.\mathrm{H}-\mathrm{C}_{5} \mathrm{H}_{8} \mathrm{O}_{4}\right]^{-}, m / z 637.43\left[\mathrm{M}-\mathrm{H}-\mathrm{C}_{5} \mathrm{H}_{8} \mathrm{O}_{4}-\mathrm{C}_{6} \mathrm{H}_{10} \mathrm{O}_{5}\right]^{-}$, and $m / z$ 475.38 [M-H- $\left.\mathrm{C}_{5} \mathrm{H}_{8} \mathrm{O}_{4}-2 \mathrm{C}_{6} \mathrm{H}_{10} \mathrm{O}_{5}\right]^{-}$, suggesting $\mathbf{C 1 9}$ is notoginsenoside $\mathrm{R} 1$ as confirmed by the reference standard. The quasi-molecular ion at $\mathrm{m} / z .1031 .5436[\mathrm{M}-\mathrm{H}]^{-}$and three diagnostic ions at $m / z$ 987.55 [M-H-44.01] $]^{-}, m / z 945.54$ [MH-86.00] $]^{-}$, and $m / z, 475.38\left[\mathrm{C}_{30} \mathrm{H}_{51} \mathrm{O}_{4}\right]^{-}$indicated that $\mathbf{C 4 4}$ is a PPT ginsenoside with a malonyl group. Also, the sequent losses of Rha, Glc, and Glc after loss of malonyl group $\left(\mathrm{C}_{3} \mathrm{H}_{2} \mathrm{O}_{3}\right)$ were inferred based on the fragment ions at $\mathrm{m} / \mathrm{z}$ $945.54\left[\mathrm{M}-\mathrm{H}-\mathrm{C}_{3} \mathrm{H}_{2} \mathrm{O}_{3}\right]^{-}, \mathrm{m} / z 799.48\left[\mathrm{M}-\mathrm{H}-\mathrm{C}_{3} \mathrm{H}_{2} \mathrm{O}_{3}-\right.$ $\left.\mathrm{C}_{6} \mathrm{H}_{10} \mathrm{O}_{4}\right]^{-}, m / z 637.43\left[\mathrm{M}-\mathrm{H}-\mathrm{C}_{3} \mathrm{H}_{2} \mathrm{O}_{3}-\mathrm{C}_{6} \mathrm{H}_{10} \mathrm{O}_{4}-\mathrm{C}_{6} \mathrm{H}_{10} \mathrm{O}_{5}\right]^{-}$, and $m / z, 475.38\left[\mathrm{M}-\mathrm{H}-\mathrm{C}_{3} \mathrm{H}_{2} \mathrm{O}_{3}-\mathrm{C}_{6} \mathrm{H}_{10} \mathrm{O}_{4}-2 \mathrm{C}_{6} \mathrm{H}_{10} \mathrm{O}_{5}\right]$, which led to chemical structure determination for $\mathbf{C 4 4}$ depicted in Fig. 5D. C162, C173, C178, and $\mathbf{C 1 9 5}$ were described as PPD-type ginsenosides on the account of the featured ion at $\mathrm{m} / \mathrm{z} 459.38\left[\mathrm{C}_{30} \mathrm{H}_{51} \mathrm{O}_{3}\right]^{-}$. Their quasi-molecular ion at $\mathrm{m} / \mathrm{z}$ $1149.61[\mathrm{M}-\mathrm{H}]^{-}$and the fragment ion at $m / z 1107.60[\mathrm{M}-\mathrm{H}-$ $\left.\mathrm{C}_{2} \mathrm{H}_{2} \mathrm{O}\right]^{-}$were suggestive of an acetyl group. The successive elimination of Glc was deduced by fragment ions at $\mathrm{m} / \mathrm{z}$ $945.52\left[\mathrm{M}-\mathrm{H}-\mathrm{C}_{2} \mathrm{H}_{2} \mathrm{O}-\mathrm{C}_{6} \mathrm{H}_{10} \mathrm{O}_{5}\right]^{-}, m / z, 783.49\left[\mathrm{M}-\mathrm{H}-\mathrm{C}_{2} \mathrm{H}_{2} \mathrm{O}-\right.$ $\left.2 \mathrm{C}_{6} \mathrm{H}_{10} \mathrm{O}_{5}\right]^{-}, \mathrm{m} / \mathrm{z} 621.44\left[\mathrm{M}-\mathrm{H}-\mathrm{C}_{2} \mathrm{H}_{2} \mathrm{O}-3 \mathrm{C}_{6} \mathrm{H}_{10} \mathrm{O}_{5}\right]^{-}$, and $\mathrm{m} / \mathrm{z}$ $459.38\left[\mathrm{M}-\mathrm{H}-\mathrm{CH}_{2} \mathrm{CO}-4 \mathrm{C}_{6} \mathrm{H}_{10} \mathrm{O}_{5}\right]^{-}$. It was deduced that peak C170 was an OA-type ginsenosides according to the characteristic ion at $\mathrm{m} / \mathrm{z}$ 455.35. The quasi-molecular ion at $\mathrm{m} / \mathrm{z}$ $793.44[\mathrm{M}-\mathrm{H}]^{-}$and its fragment ions at $m / z 631.38[\mathrm{M}-\mathrm{H}-$ $\left.\mathrm{C}_{6} \mathrm{H}_{10} \mathrm{O}_{5}\right]^{-}$and $m / z, 455.35\left[\mathrm{M}-\mathrm{H}-\mathrm{C}_{6} \mathrm{H}_{10} \mathrm{O}_{5}-\mathrm{C}_{6} \mathrm{H}_{8} \mathrm{O}_{6}\right]^{-}$were indicative of a Glc and a GluA connecting to OA in C170. Similarly, C2 was tentatively characterized as OT-6Glc(2,1)Rha-Glc by the $[\mathrm{M}-\mathrm{H}]^{-}$ions at $m / z, 961.54$ and fragment ions at $m / z 799.48\left[\mathrm{M}-\mathrm{H}-\mathrm{C}_{6} \mathrm{H}_{10} \mathrm{O}_{5}\right]^{-}, m / z 653.42[\mathrm{M}-\mathrm{H}-$ $\left.\mathrm{C}_{6} \mathrm{H}_{10} \mathrm{O}_{5}-\mathrm{C}_{6} \mathrm{H}_{10} \mathrm{O}_{4}\right]^{-}$, and $\mathrm{m} / z$ 491.37 [M-H- $\mathrm{C}_{6} \mathrm{H}_{10} \mathrm{O}_{5^{-}}$ $\left.\mathrm{C}_{6} \mathrm{H}_{10} \mathrm{O}_{4}-\mathrm{C}_{6} \mathrm{H}_{10} \mathrm{O}_{5}\right]^{-}$with corresponding losses of Glc, GlcRha, and Glc-Rha-Glc respectively [20]. Sapogenins of licorice saponins are mainly pentacyclic triterpenes. For example, glycyrrhizic acid, identified through comparison with the standard, gave base-peak ion at $m / z$ 821.40, which was assigned a formula of $\mathrm{C}_{42} \mathrm{H}_{62} \mathrm{O}_{16}$. As depicted in Fig. 5E, the possible fragmentation pathways of glycyrrhizic acid were deduced via the typical ions at $m / z 759.3916\left[\mathrm{M}-\mathrm{H}-\mathrm{CH}_{2} \mathrm{O}_{3}\right]^{-}, \mathrm{m} / \mathrm{z}$ $645.3618\left[\mathrm{M}-\mathrm{H}-\mathrm{C}_{6} \mathrm{H}_{8} \mathrm{O}_{6}\right]^{-}, m / z 469.3315\left[\mathrm{M}-\mathrm{H}-2 \mathrm{C}_{6} \mathrm{H}_{8} \mathrm{O}_{6}\right]^{-}$, and $\mathrm{m} / \mathrm{z}, 351.0568\left[\mathrm{C}_{12} \mathrm{H}_{15} \mathrm{O}_{12}\right]^{-}$. The potential structures of C73, C94, C154, and C210 were also deduced based on the characteristic ions and main fragmentation pathways as other saponins in MS-network, which might also serve as novel agents (see ESM Fig. S4C-F).

\section{Other components}

Despite the efficiency and convenience of MS-network in rapid qualitative analysis, this approach is not always wellsuited for identifying all types of components in TCM formulae. Thus, 35 other components, including coumarins, phenols, phenyl derivatives, and similar, were characterized via their $\mathrm{MS}^{1}$ and featured fragment ions as well as corresponding losses. Taking D30 as a case, it gave a precursor ion at $\mathrm{m} / \mathrm{z}$ $367.12[\mathrm{M}-\mathrm{H}]^{-}$, which was assigned a formula of $\mathrm{C}_{21} \mathrm{H}_{20} \mathrm{O}_{6}$. The diagnostic ions at $m / z 352.10\left[\mathrm{M}-\mathrm{H}-\mathrm{CH}_{3}\right]^{-}, \mathrm{m} / z 339.12$ $[\mathrm{M}-\mathrm{H}-\mathrm{CO}]^{-}, m / z, 337.07\left[\mathrm{M}-\mathrm{H}-\mathrm{C}_{2} \mathrm{H}_{6}\right]^{-}, m / z, 324.10[\mathrm{M}-\mathrm{H}-$ $\left.\mathrm{CO}-\mathrm{CH}_{3}\right]^{-}$, and $\mathrm{m} / \mathrm{z}, 309.04\left[\mathrm{M}-\mathrm{H}-\mathrm{C}_{4} \mathrm{H}_{10}\right]^{-}$were ascribed to different fragments, as depicted in Fig. $4 \mathrm{f}$ and $5 \mathrm{~F}$, which were consistent with those of previously reported glycycoumarin [41]. Comparing the exact molecular weights and MS or MS/MS spectra with those in references and online databases led to the identification of other compounds, as shown in Table S5 (see ESM2).

\section{Quantitative analysis of chemical constituents in SJZD by UHPLC-QTRAP ${ }^{\oplus}$-MS/MS}

To further understand the contents of potential bioactive constituents, a UHPLC-QTRAP ${ }^{\circledR}$-MS/MS was developed for method validation and quantitative analysis of 19 compounds.

\section{Optimization of UHPLC-MS/MS conditions}

To obtain good chromatographic behaviors and appropriate ionization efficiency, different mobile phase systems (methanol/water, acetonitrile-water, methanol-acid aqueous solution, and acetonitrile-acid aqueous solution) were compared, revealing that acetonitrile $0.1 \%$ aqueous formic acid was the best choice, which showed a better response.

The MRM mode was used for quantification. In order to select the appropriate fragmentation mode of $\mathrm{m} / \mathrm{z}(\mathrm{Q1})$ and $\mathrm{m} / \mathrm{z}$ $(Q 3)$, these reference standards were directly tested in the mass spectrum, and the scanning mass spectra of the product ions were recorded. In addition, the MRM conditions were also optimized for the analytes as shown in Table S1 (see ESM).

\section{Method validation}

Linearity equations were obtained by plotting corresponding peak areas $(y)$ versus concentrations $(x)$. Acceptable linear correlations of these 19 analytes at these conditions were confirmed by the correlation coefficients $\left(R^{2}, 0.9971-0.9999\right)$. As shown in Table 1, the intra-day and inter-day precision of this method calculated as relative standard deviation (RSD) were within the range of $0.49-3.01 \%$. The repeatability range was $1.06-3.51 \%$ and the RSDs of stability test of the samples stored at $8{ }^{\circ} \mathrm{C}$ were ranging from 1.11 to $3.50 \%$ within 24-h analysis. The mean average recovery rates for the 19 analytes ranged from 93.05 to $104.34 \%$, with RSD values of 0.69 to $5.56 \%$. Collectively, the optimized phase and MS methods exhibited good precision, reproducibility, stability, and recovery. 


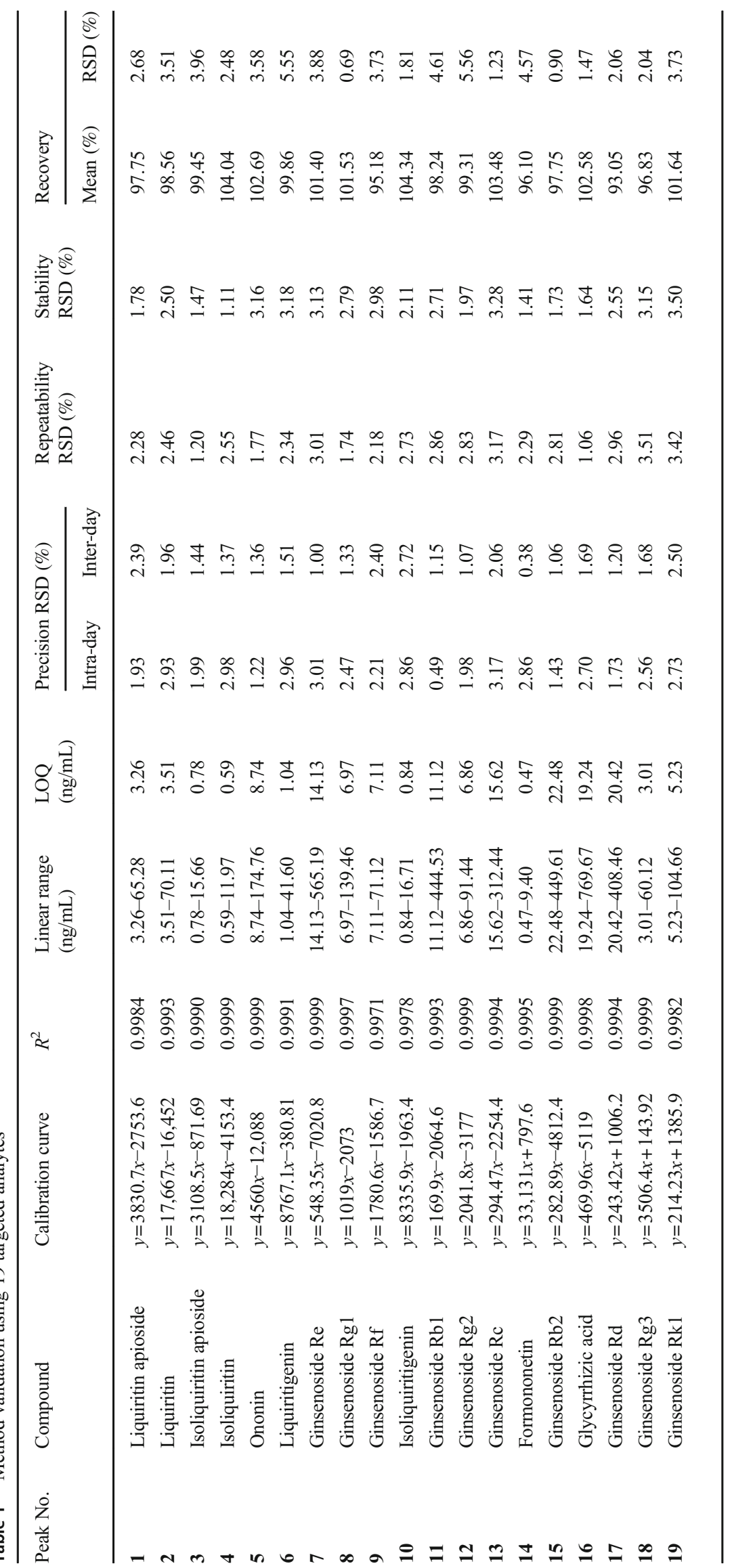




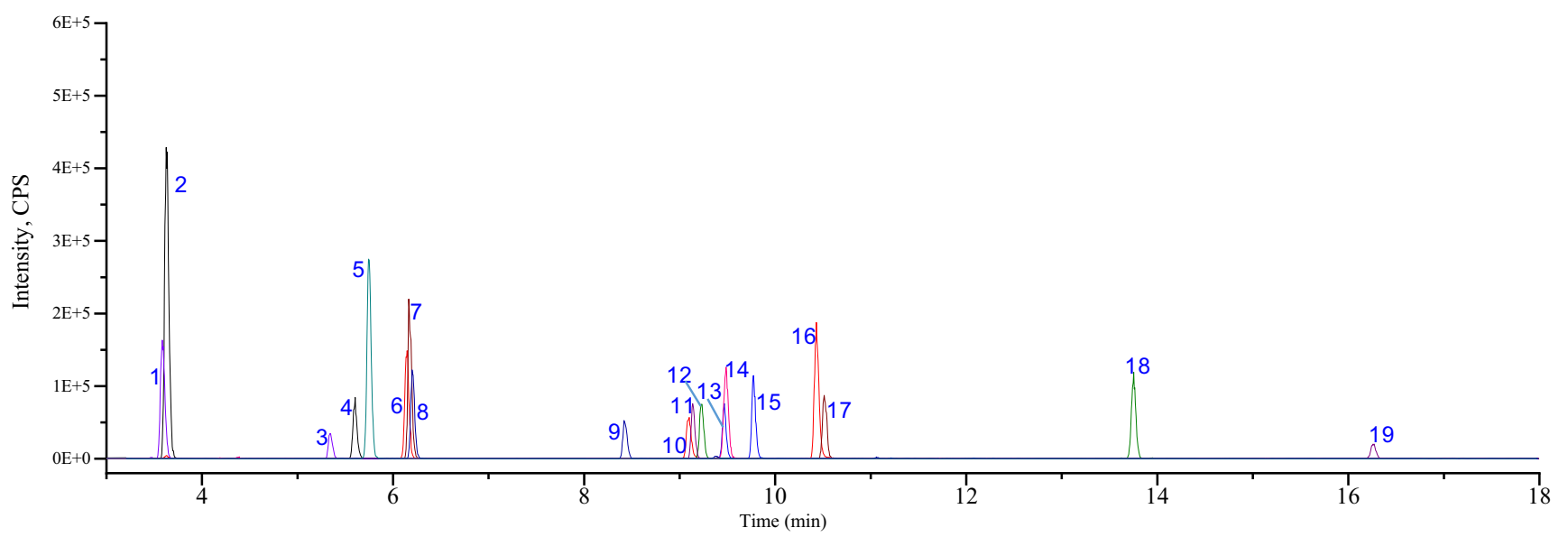

Fig. 6 Representative MRM chromatograms of 19 analytes from standard solutions on UHPLC-QTRAP ${ }^{\circledR}$-MS/MS system $(\mathbf{1}$, liquiritin apioside; $\mathbf{2}$, liquiritin; $\mathbf{3}$, isoliquiritin apioside; $\mathbf{4}$, isoliquiritin; $\mathbf{5}$, ononin; 6, liquiritigenin; 7, ginsenoside Re; 8 , ginsenoside Rg1; 9, ginsenoside

\section{Contents of the selected compounds in SJZD}

Since the main ingredients of NPSs in SJZD are saponins and flavonoids, the newly developed UHPLC-QTOF-MS/MS was employed in the SJZD, and the components were sorted according to the peak response intensity and peak area in the mass spectrum therewith. After combination with the in vivo

Table 2 The contents (mg/g) of the 19 constituents in SJZD

\begin{tabular}{lllll}
\hline Peak No. & \multicolumn{3}{l}{ Content $^{\mathrm{a}}(\mathrm{mg} / \mathrm{g})(n=3)$} & \\
\cline { 2 - 5 } & Sample 1 & Sample 2 & Sample 3 & Mean $\pm \mathrm{SD}$ \\
\hline $\mathbf{1}$ & 1.796 & 1.714 & 1.818 & $1.776 \pm 0.055$ \\
$\mathbf{2}$ & 0.614 & 0.644 & 0.625 & $0.628 \pm 0.016$ \\
$\mathbf{3}$ & 0.162 & 0.167 & 0.175 & $0.168 \pm 0.007$ \\
$\mathbf{4}$ & 0.082 & 0.097 & 0.091 & $0.090 \pm 0.008$ \\
$\mathbf{5}$ & 0.129 & 0.131 & 0.142 & $0.134 \pm 0.007$ \\
$\mathbf{6}$ & 0.021 & 0.027 & 0.024 & $0.024 \pm 0.003$ \\
$\mathbf{7}$ & 0.618 & 0.634 & 0.656 & $0.636 \pm 0.019$ \\
$\mathbf{8}$ & 0.275 & 0.291 & 0.277 & $0.281 \pm 0.009$ \\
$\mathbf{9}$ & 0.169 & 0.179 & 0.177 & $0.175 \pm 0.005$ \\
$\mathbf{1 0}$ & 0.010 & 0.013 & 0.011 & $0.011 \pm 0.002$ \\
$\mathbf{1 1}$ & 0.651 & 0.679 & 0.689 & $0.673 \pm 0.020$ \\
$\mathbf{1 2}$ & 0.167 & 0.171 & 0.184 & $0.174 \pm 0.009$ \\
$\mathbf{1 3}$ & 0.478 & 0.509 & 0.492 & $0.493 \pm 0.016$ \\
$\mathbf{1 4}$ & 0.019 & 0.022 & 0.016 & $0.019 \pm 0.003$ \\
$\mathbf{1 5}$ & 0.515 & 0.519 & 0.499 & $0.511 \pm 0.011$ \\
$\mathbf{1 6}$ & 1.789 & 1.682 & 1.632 & $1.701 \pm 0.080$ \\
$\mathbf{1 7}$ & 0.597 & 0.633 & 0.603 & $0.611 \pm 0.019$ \\
$\mathbf{1 8}$ & 0.057 & 0.064 & 0.062 & $0.061 \pm 0.004$ \\
$\mathbf{1 9}$ & 0.159 & 0.138 & 0.141 & $0.146 \pm 0.011$ \\
\hline $\mathbf{9}$ & & & &
\end{tabular}

${ }^{\text {a }}$ Calculated as crude drug
Rf; 10, isoliquiritigenin; 11, ginsenoside $\mathrm{Rb} 1 ; \mathbf{1 2}$, ginsenoside $\mathrm{Rg} 2 ; \mathbf{1 3}$, ginsenoside Rc; 14, formononetin; 15, ginsenoside Rb2; 16, glycyrrhizic acid; 17, ginsenoside Rd; 18, ginsenoside Rg3; 19, ginsenoside Rk1)

and in vitro activity reports of each single herb mainly invoving anti-inflammatory, neuroprotective, and immunomodulatory effects, which related to the traditonal efficiency of SJZD [42-50], 19 components were screened out for quantification. The established analytical approach in UHPLCQTRAP $^{\circledR}$-MS/MS within 20 min was applied for the simultaneous quantification of these compounds. Representative chromatograms for reference standards and SJZD samples are shown in Fig. 6 and Fig. S5 (see ESM). As shown in Table 2, the quantitative results showed that liquiritin apioside and glycyrrhizic acid had the highest content of all the 19 ingredients, and each content of liquiritin, ginsenoside Re, ginsenoside $\mathrm{Rb} 1$, ginsenoside $\mathrm{Rb} 2$, and ginsenoside $\mathrm{Rd}$ was greater than $0.5 \mathrm{mg} / \mathrm{g}$. Considering their high content and multiple activity reports, these components might have the potential to be developed as quality markers for SJZD. In addition, the content of these selected constituents in licorice and ginseng in SJZD was not lower than $4.551 \mathrm{mg} / \mathrm{g}$ and $3.761 \mathrm{mg} / \mathrm{g}$, respectively.

\section{Discussion}

UHPLC-QTOF-MS/MS has been widely used in the qualitative analysis of TCM, but it still has many limitations. The first limitation is related to the peak capacity of UHPLC separation, which is proportional to the average resolution $[51,52]$. The second one is that many trace components could not be identified due to the differences in the ingredients' content and ionization efficiency in the prescription. As Giddings proposed, peak capacity has become the most important separation ability indicator in multi-dimensional separation [51, 52]. Compared to 1DLC-MS, 2DLC-MS can increase the elution system's peak capacity and the ionization efficiency of trace components greatly, thus is considered a powerful tool for the 
Fig. 7 A summary of possible sources (a), classification (b), components of Fr.1-5 (c), and the retention time and quasimolecular ions (d) of these compounds identified from SJZD. Abbreviation notes: $\mathrm{G}$ represented Ginseng Radix et Rhizoma; AM represented Atractylodes Macrocephalae Rhizoma; P represented Poria; Gly represented Glycyrrhizae Radix et Rhizoma
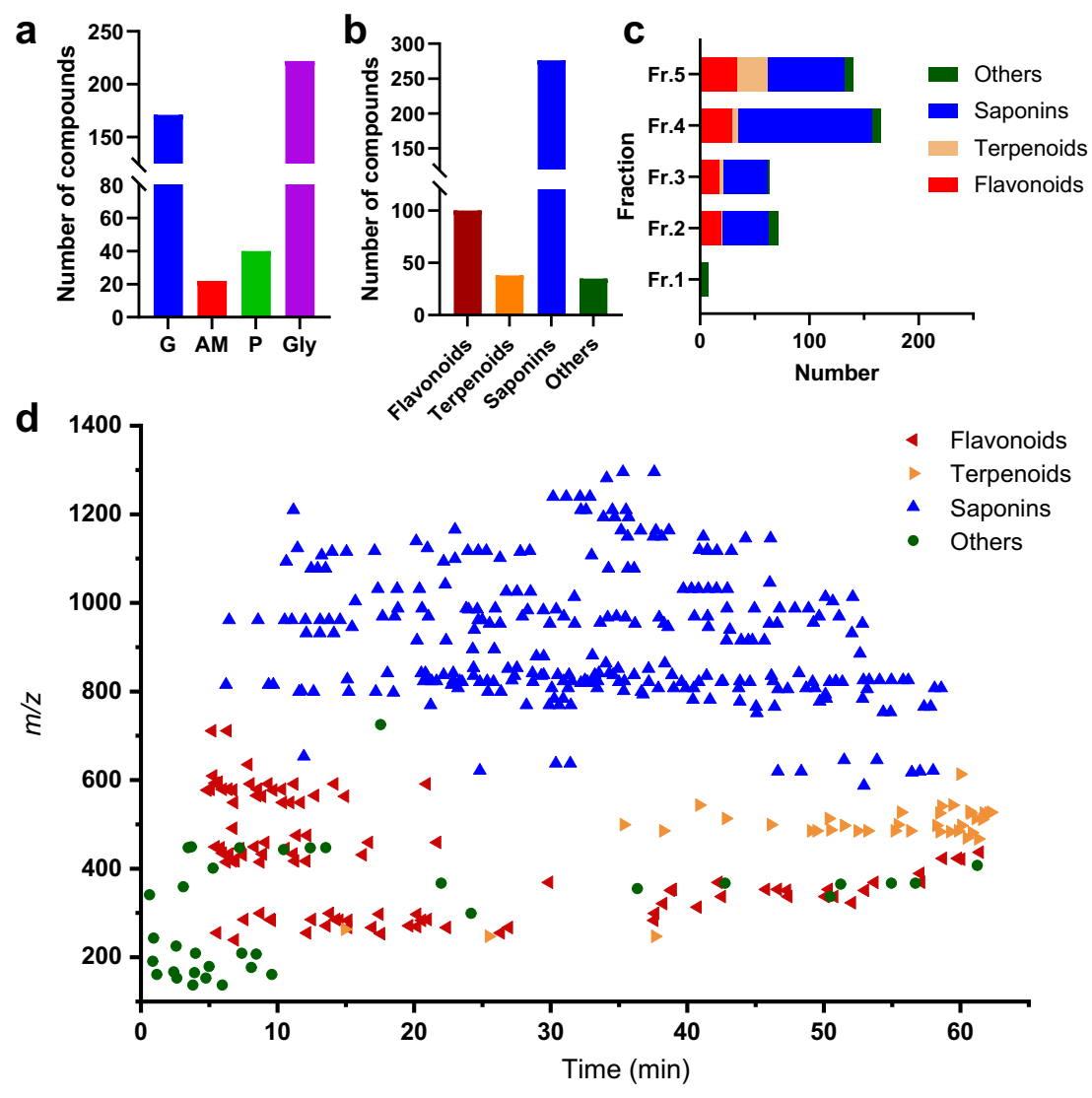

separation and identification of complex components of TCM [18]. Due to the poor separation of hydrophilic compounds, such as flavonoids and saponins on the HILIC column, RPLC $\times$ RPLC was chosen for the separation of NPSs in SJZD. Considering that the ${ }^{2} \mathrm{D}$ column, which is directly connected to the MS, has an important role in the final separation and analysis, in the present study, we compared four columns used for ${ }^{2} \mathrm{D}$ separation based on column efficiency and peak response intensity. As calculated with Neue's method [53], the theoretical peak capacity of Waters BEH C18, ZORBAX Eclipse Plus C18, Agilent Poroshell 120 EC-C18, and ZORBAX SB-C8 was 107, 126, 133, and 113, respectively. Both Waters BEH C18 and ZORBAX Eclipse Plus C18 were found to exhibit better column efficiency, with the complete separation of liquiritin and liquiritin apioside (see ESM Fig. S2). ZORBAX Eclipse Plus C18 showed higher peak response intensity than the other three, and was finally selected as the ${ }^{2} \mathrm{D}$ column. The qualitative results indicated that the developed offline RPLC $\times$ RPLC-MS/MS exhibited excellent performance at separation and ionization of the secondary metabolites in SJZD.

Along with the larger flux of quasi-molecular ions brought about by 2DLC-MS, optimal characterization has become another critical issue. It is necessary to develop an effective and rapid method for comprehensive structural analysis of TCM components to assign as many as detected ions. MS-network analysis pattern utilizing the structural correlation characteristics of secondary metabolites in herbs provides an effective solution for the rapid and accurate identification of components and even its metabolites in vivo [21]. In this work, the MS-networks of SJZD were established based on the molecular formulas of the basic skeletons and structural correlations in flavonoids, terpenoids, and saponins. Additionally, we also considered the issue of isomers in the process of MS-network establishment. For example, in MS-network of flavonoids, the molecular formulas in $\mathrm{A}_{1}$ (see Fig. 2a), derived by two core skeletons, actually contain different isomers. The molecular structure of the basic skeleton was determined by a quasimolecular ion screening through MS-network firstly and then the probable structure or its isomers were finally determined by the analysis of corresponding losses in the MS/MS in detail, which made the qualitative analysis easier and faster.

In this study, 449 ingredients were identified and tentatively characterized, including 6 potential new compounds. Compared with previous reports, this study reported the largest number of ingredients described in SJZD. The possible sources of these compounds were assigned based on phytochemical reports of four herbs and analysis of the structures shown in Tables S2-5 (see ESM2) and Fig. 7a, b. The components of different fractions were further analyzed. As depicted in Fig. 7c, most components were collected in Fr.4 and Fr.5, with more distribution of saponins. Additionally, the 
terpenoids were mainly collected in Fr.5, probably due to the low polarity. A summary of the elution order and quasimolecular ions of these four classes of compounds characterized in SJZD was also illustrated. As shown in Fig. 7d, the four types of components were clearly distinguished in the distribution of the $\mathrm{m} / \mathrm{z}$ dimension, among which the average $\mathrm{m} / \mathrm{z}$ of other types of components in SJZD was the lowest. The $\mathrm{m} / \mathrm{z}$ values of the majority of flavonoids and terpenoids were below 600 , while those of saponin ingredients were above 600. In addition, the retention behavior of the flavonoids and the triterpenoids was mainly located in both ends of the elution order, while the saponins were distributed throughout the whole elution process, which might provide essential information for studies on other TCMs.

In this quantitative study, 19 representative compounds were finally selected based on the content of ingredients in the original decoction as well as considering related activity reports. Considering that some compounds responded better in MS after adding formic acid into the mobile phase, and the formic acid peaks of partial compounds were also selected as quantitative ions, the acetonitrile-acid aqueous solution mobile phase was ultimately chosen as the mobile phase. Compared with previous quantification research on SJZD, more ingredients were quantified [14, 54]. To the best of our knowledge, this is the first application of TQ-MS/MS for the determination of the content of NPSs in SJZD.

\section{Conclusion}

In the present study, we established and optimized a MSnetwork-based offline 2DLC-QTOF-MS/MS for rapid identification of secondary metabolites that was successfully applied for comprehensive characterization of the nonpolysaccharides in SJZD. As the major secondary metabolites of SJZD are saponins, flavonoids, and terpenoids, the RPLC $\times$ RPLC was developed for the separation and characterization with the optimal LC and MS conditions. In addition, the MSnetworks of flavonoids, terpenoids, and saponins in SJZD were established based on basic skeletons of different types of compounds and their structural correlations, which remarkably expanded the in-house chemical library and accelerated qualitative analysis. As a result, a total of 449 components were definitively or tentatively identified, of which 6 were deduced as potentially novel ones. These components were further analyzed and summarized in terms of structure types, distribution in $\mathrm{m} / \mathrm{z}$ dimension and different fractions, and elution order, thus providing essential information for more advanced analysis of flavonoids, terpenoids, and saponins in SJZD and other TCMs. Meanwhile, based on peak response intensity and activity reports of in vivo and in vitro, 19 representative ingredients with characterized structures were selected and then quantified by a developed method in UHPLC-QTRAP ${ }^{\circledR}$-MS/MS. Our results showed that liquiritin apioside, glycyrrhizic acid, liquiritin, ginsenoside $\mathrm{Re}$, ginsenoside $\mathrm{Rb} 1$, ginsenoside $\mathrm{Rb} 2$, and ginsenoside Rd, each with content greater than $0.5 \mathrm{mg} / \mathrm{g}$, may have the potential to be developed as quality markers of SJZD. These results suggested that the integrated strategy we developed is an effective approach for the comprehensive profiling of TCM recipes as well as the discovery of potential new agents.

Abbreviations SJZD, Sijunzi decoction; TCM, Traditional Chinese medicine; NPS, Non-polysaccharide; 2DLC-QTOF-MS/MS, Two-dimensional liquid chromatography coupled with quadrupole time-offlight tandem mass spectrometry; UHPLC-QTRAP ${ }^{\circledR}-$ MS/MS, Ultrahigh-performance liquid chromatography coupled with triplequadrupole linear ion-trap tandem mass spectrometry; 1D, One-dimensional; 2D, Two-dimensional; ${ }^{1} \mathrm{D}$, First-dimensional; ${ }^{2} \mathrm{D}$, Second-dimensional; LC, Liquid chromatography; LC-MS, Liquid chromatographymass spectrometry; UHPLC-QTOF-MS/MS, Ultra-performance liquid chromatography coupled with quadrupole time-of-flight tandem mass spectrometry; 1DLC, One-dimensional liquid chromatography; 2DLC, Two-dimensional liquid chromatography; UHPLC-TQ-MS, Ultra-highperformance liquid chromatography coupled with triple-quadrupole tandem mass spectrometry; MRM, Multiple reaction monitoring; RPLC $\times$ RPLC-QTOF-MS, Reversed-phase $\times$ reversed-phase two-dimensional liquid chromatography quadrupole time-of-flight mass spectrometry; GS1, Nebulizer gas; GS2, Auxiliary gas; BPC, Base peak intensity chromatogram; Glc, Glucose; Apio, Apiose; Rha, Rhamnose; Xyl, Xylose; $\operatorname{Ara}(f)$, Furan-arabinose; Ara $(p)$, Pyran-arabinose; GluA, Glucuronic acid; PPD, Protopanaxadiol; PPT, Protopanaxatriol; OA, Oleanolic acid; OT, Octillol; Mal, Malonyl

Supplementary Information The online version contains supplementary material available at https://doi.org/10.1007/s00216-021-03302-x.

Funding This work was supported by grants from the National Natural Science Foundation of China (No. 81973441).

\section{Declarations}

Conflict of interest The authors declare no competing interests.

\section{References}

1. Notice regarding the issuance of a new coronavirus pneumonia diagnosis and treatment plan (for trial version 6, 7, and 8). http:// www.gov.cn/zhengce/zhengceku/2020-02/19/content_5480948. htm; http://www.gov.cn/zhengce/zhengceku/2020-03/04/content 5486705.htm; http://www.gov.cn/zhengce/zhengceku/2020-08/19/ content_5535757.htm. Accessed 3 Sept 2020.

2. Tian G, Wu C, Li J, Liang B, Zhang F, Fan X, et al. Network pharmacology based investigation into the effect and mechanism of modified Sijunzi decoction against the subtypes of chronic atrophic gastritis. Pharmacol Res. 2019;144:158-66. https://doi.org/10. 1016/j.phrs.2019.04.012.

3. Chen CM, Lee CY, Lin PJ, Hsieh CL, Shih HC. Evaluation on the pharmacological effect of traditional Chinese medicine SiJunZiTang on stress-induced peptic ulcers. Evid Based 
Complement Alternat Med. 2013;2013:186076. https://doi.org/10. 1155/2013/186076.

4. Shi Y, Zhu H, Li R, Wang D, Zhu Y, Hu L, et al. Effect of polysaccharides from Sijunzi decoction on $\mathrm{Ca}^{2+}$ related regulators during intestinal mucosal restitution. Phytomedicine. 2019;58:152880. https://doi.org/10.1016/j.phymed.2019.152880.

5. Huang C, Zhu Z, Cao X, Chen X, Fu Y, Chen Z, et al. A pectic polysaccharide from Sijunzi decoction promotes the antioxidant defenses of SW480 cells. Molecules. 2017;22(8):1341. https://doi. org/10.3390/molecules22081341.

6. Ye L, Gong J, Wang Y, Sun X. Pharmacological activities of Sijunzi decoction which are related to its antioxidant properties. J Chem. 2014;2014:1-10. https://doi.org/10.1155/2014/278318.

7. Wang R, Ji Y, Peng Y, Li X. Preliminary characterization of a homogeneous polysaccharide with anticomplement activity from Sijunzi decoction. J Chem. 2018;2018:1-8. https://doi.org/10. 1155/2018/8642653

8. Gao B, Wang R, Peng Y, Li X. Effects of a homogeneous polysaccharide from Sijunzi decoction on human intestinal microbes and short chain fatty acids in vitro. J Ethnopharmacol. 2018;224:46573. https://doi.org/10.1016/j.jep.2018.06.006.

9. Wu B, Xuan ZR. Progress in research on applying Sijunzi decoction in treating digestive malignant tumor. Chin J Integr Med. 2007;13(2):156-9. https://doi.org/10.1007/s11655-007-0156-Z.

10. Jia J, Qin Y, Zhang L, Guo C, Wang Y, Yue X, et al. Sijunzi decoction-treated rat serum induces apoptosis of side population cells in gastric carcinoma. Exp Ther Med. 2018;15(2):1718-27. https://doi.org/10.3892/etm.2017.5560.

11. Wang R, Peng Y, Meng H, Li X. Protective effect of polysaccharides fractions from Sijunzi decoction in reserpine-induced spleen deficiency rats. RSC Adv. 2016;6(65):60657-65. https://doi.org/ 10.1039/c6ra06361f.

12. Liu Y, Yang J, Cai Z. Chemical investigation on Sijunzi decoction and its two major herbs Panax ginseng and Glycyrrhiza uralensis by LC/MS/MS. J Pharm Biomed Anal. 2006;41(5):1642-7. https:// doi.org/10.1016/j.jpba.2006.02.033.

13. Wang Y, He S, Cheng X, Lu Y, Zou Y, Zhang Q. UPLC-Q-TOFMS/MS fingerprinting of traditional Chinese formula SiJunZiTang. J Pharm Biomed Anal. 2013;80:24-33. https://doi.org/10.1016/j. jpba.2013.02.021.

14. Huang W, Hou J, Wang M, Peng C, Peng Y, Li X. Qualitative and quantitative analysis of constituents in Si-jun-zi Tang decoction by HPLC-DAD-ELSD and HPLC-ESI-MS. Curr Pharm Anal. $2015 ; 11$ (2): 115-23. https://doi.org/10.2174/ 1573412911666141208231409.

15. Guan Z, Wang M, Cai Y, Yang H, Zhao M, Zhao C. Rapid characterization of the chemical constituents of Sijunzi decoction by UHPLC coupled with Fourier transform ion cyclotron resonance mass spectrometry. J Chromatogr B Anal Technol Biomed Life Sci. 2018;1086:11-22. https://doi.org/10.1016/j.jchromb.2018.04. 009.

16. Qiao X, Song W, Ji S, Li YJ, Wang Y, Li R, et al. Separation and detection of minor constituents in herbal medicines using a combination of heart-cutting and comprehensive two-dimensional liquid chromatography. J Chromatogr A. 2014;1362:157-67. https://doi. org/10.1016/j.chroma.2014.08.038.

17. Hu X, Liu M, Liu Y, Zhang J, Tang Y, Pei H, et al. A two-step approach for systematic identification and quality evaluation of wild and introduced Anemone flaccida Fr. Schmidt (Di Wu) based on DNA barcode and UPLC-QTOF-MS/MS. Anal Bioanal Chem. 2020;412(8):1807-16. https://doi.org/10.1007/s00216-020-02426w.

18. Dugo P, Cacciola F, Kumm T, Dugo G, Mondello L. Comprehensive multidimensional liquid chromatography: theory and applications. J Chromatogr A. 2008;1184(1):353-68. https:// doi.org/10.1016/j.chroma.2007.06.074.
19. Zuo T, Zhang C, Li W, Wang H, Hu Y, Yang W, et al. Offline twodimensional liquid chromatography coupled with ion mobilityquadrupole time-of-flight mass spectrometry enabling fourdimensional separation and characterization of the multicomponents from white ginseng and red ginseng. J Pharm Anal. 2019. https://doi.org/10.1016/j.jpha.2019.11.001.

20. Yang WZ, Ye M, Qiao X, Liu CF, Miao WJ, Bo T, et al. A strategy for efficient discovery of new natural compounds by integrating orthogonal column chromatography and liquid chromatography/ mass spectrometry analysis: its application in Panax ginseng, Panax quinquefolium and Panax notoginseng to characterize 437 potential new ginsenosides. Anal Chim Acta. 2012;739:56-66. https://doi.org/10.1016/j.aca.2012.06.017.

21. Li Z, Guo X, Cao Z, Liu X, Liao X, Huang C, et al. New MS network analysis pattern for the rapid identification of constituents from traditional Chinese medicine prescription Lishukang capsules in vitro and in vivo based on UHPLC/Q-TOF-MS. Talanta. 2018;189:606-21. https://doi.org/10.1016/j.talanta.2018.07.020.

22. Wolfender JL, Marti G, Thomas A, Bertrand S. Current approaches and challenges for the metabolite profiling of complex natural extracts. J Chromatogr A. 2015;1382:136-64. https://doi.org/10. 1016/j.chroma.2014.10.091.

23. Zheng R, Xu H, Wang W, Zhan R, Chen W. Simultaneous determination of aflatoxin B1, B2, G1, G2, ochratoxin A, and sterigmatocystin in traditional Chinese medicines by LC-MSMS. Anal Bioanal Chem. 2014;406(13):3031-9. https://doi.org/ 10.1007/s00216-014-7750-7.

24. Wishart DS, Feunang YD, Marcu A, Guo AC, Liang K, VazquezFresno R, et al. HMDB 4.0: the human metabolome database for 2018. Nucleic Acids Res. 2018;46(D1):D608-D17. https://doi.org/ 10.1093/nar/gkx1089.

25. Horai H, Arita M, Kanaya S, Nihei Y, Ikeda T, Suwa K, et al. MassBank: a public repository for sharing mass spectral data for life sciences. J Mass Spectrom. 2010;45(7):703-14. https://doi.org/ 10.1002/jms. 1777 .

26. Agency EM. Guideline on bioanalytical method validation. European Medicines Agency. 2011 (Accessed on August 2018). https://www.ema.europa.eu/en/documents/scientific-guideline/ guideline-bioanalytical-method-validation en.pdf.

27. Ji S, Wang S, Xu H, Su Z, Tang D, Qiao X, et al. The application of on-line two-dimensional liquid chromatography (2DLC) in the chemical analysis of herbal medicines. J Pharm Biomed Anal. 2018;160:301-13. https://doi.org/10.1016/j.jpba.2018.08.014.

28. Qiao X, Song W, Ji S, Wang Q, Guo DA, Ye M. Separation and characterization of phenolic compounds and triterpenoid saponins in licorice (Glycyrrhiza uralensis) using mobile phase-dependent reversed-phasexreversed-phase comprehensive two-dimensional liquid chromatography coupled with mass spectrometry. J Chromatogr A. 2015;1402:36-45. https://doi.org/10.1016/j. chroma.2015.05.006.

29. Li D, Schmitz OJ. Comprehensive two-dimensional liquid chromatography tandem diode array detector (DAD) and accurate mass QTOF-MS for the analysis of flavonoids and iridoid glycosides in Hedyotis diffusa. Anal Bioanal Chem. 2015;407(1):231-40. https:// doi.org/10.1007/s00216-014-8057-4.

30. Xie Y, Zhao W, Zhou T, Fan G, Wu Y. An efficient strategy based on MAE, HPLC-DAD-ESI-MS/MS and 2D-prep-HPLC-DAD for the rapid extraction, separation, identification and purification of five active coumarin components from Radix Angelicae Dahuricae. Phytochem Anal. 2010;21(5):473-82. https://doi.org/ 10.1002/pca.1222.

31. Zhou W, Guo Z, Yu L, Zhou H, Shen A, Jin Y, et al. On-line comprehensive two-dimensional liquid chromatography tandem mass spectrometry for the analysis of Curcuma kwangsiensis. Talanta. 2018;186:73-9. https://doi.org/10.1016/j.talanta.2018.04. 014 . 
32. Godfrey AR, Brenton AG. Accurate mass measurements and their appropriate use for reliable analyte identification. Anal Bioanal Chem. 2012;404(4):1159-64. https://doi.org/10.1007/s00216-0126136-y.

33. Liu X, Fan X, Wang X, Liu R, Meng C, Wang C. Structural characterization and screening of chemical markers of flavonoids in Lysimachiae Herba and Desmodii Styracifolii Herba by ultra high-performance liquid chromatography quadrupole time-offlight tandem mass spectrometry based metabolomics approach. J Pharm Biomed Anal. 2019;171:52-64. https://doi.org/10.1016/j. jpba.2019.04.003.

34. Jiang Z, Wang Y, Zhu Y, Zhang L, Chai X, Jiang M, et al. Dosedependent targeted knockout methodology combined with deep structure elucidation strategies for Chinese licorice chemical profiling. J Pharm Biomed Anal. 2015;115:130-7. https://doi.org/10. 1016/j.jpba.2015.06.020.

35. Du Y, He B, Li Q, He J, Wang D, Bi K. Identification and analysis of chemical constituents and rat serum metabolites in Suan-ZaoRen granule using ultra high performance liquid chromatography quadrupole time-of-flight mass spectrometry combined with multiple data processing approaches. J Sep Sci. 2017;40(14):2914-24. https://doi.org/10.1002/jssc.201700236.

36. Zhang Y, Wang C, Yang F, Yang Z, Wang F, Sun G. UHPLC-ESIQ-TOF-MS/MS analysis, antioxidant activity combined fingerprints for quality consistency evaluation of compound liquorice tablets. RSC Adv. 2018;8(49):27661-73. https://doi.org/10.1039/ c8ra02431f.

37. Dong J, Zhu Y, Gao X, Chang Y, Wang M, Zhang P. Qualitative and quantitative analysis of the major constituents in Chinese medicinal preparation Dan-Lou tablet by ultra high performance liquid chromatography/diode-array detector/quadrupole time-of-flight tandem mass spectrometry. J Pharm Biomed Anal. 2013;80:50 62. https://doi.org/10.1016/j.jpba.2013.02.011.

38. Wang X, Li X, Peng C. Comprehensive mass spectrum analysis of two flavone-6, 8-C-di-glycosides and its application by high resolution electrospray ionization tandem mass spectroscopy in both negative and positive ion modes. China J Chin Mater Med. 2019;44(22):4880-7. https://doi.org/10.19540/j.cnki.cjcmm. 20190823.201.

39. Feng GF, Zheng Y, Sun Y, Liu S, Pi ZF, Song FR, et al. A targeted strategy for analyzing untargeted mass spectral data to identify lanostane-type triterpene acids in Poria cocos by integrating a scientific information system and liquid chromatography-tandem mass spectrometry combined with ion mobility spectrometry. Anal Chim Acta. 2018;1033:87-99. https://doi.org/10.1016/j.aca.2018.06.048.

40. Schmid C, Dawid C, Peters V, Hofmann T. Saponins from European licorice roots (Glycyrrhiza glabra). J Nat Prod. 2018;81(8):1734-44. https://doi.org/10.1021/acs.jnatprod. $8 \mathrm{~b} 00022$.

41. Zhu J, Yi X, Zhang J, Chen S, Wu Y. Chemical profiling and antioxidant evaluation of Yangxinshi tablet by HPLC-ESI-QTOF-MS/MS combined with DPPH assay. J Chromatogr B Anal Technol Biomed Life Sci. 2017;1060:262-71. https://doi.org/10. 1016/j.jchromb.2017.06.022.

42. Ji S, Li Z, Song W, Wang Y, Liang W, Li K, et al. Bioactive constituents of Glycyrrhiza uralensis (licorice): discovery of the effective components of a traditional herbal medicine. J Nat Prod. 2016;79(2):281-92. https://doi.org/10.1021/acs.jnatprod.5b00877.

43. Yang F, Chu T, Zhang Y, Liu X, Sun G, Chen Z. Quality assessment of licorice (Glycyrrhiza glabra L.) from different sources by multiple fingerprint profiles combined with quantitative analysis, antioxidant activity and chemometric methods. Food Chem. 2020;324:126854. https://doi.org/10.1016/j.foodchem.2020. 126854.

44. Zhang Y, Yang F, Zhang J, Sun G, Wang C, Guo Y, et al. Quantitative fingerprint and quality control analysis of compound liquorice tablet combined with antioxidant activities and chemometrics methods. Phytomedicine. 2019;59:152790. https:// doi.org/10.1016/j.phymed.2018.12.013.

45. Kao TC, Wu CH, Yen GC. Bioactivity and potential health benefits of licorice. J Agric Food Chem. 2014;62(3):542-53. https://doi.org/ 10.1021/jf404939f.

46. Zhai KF, Duan H, Cui CY, Cao YY, Si JL, Yang HJ, et al. Liquiritin from Glycyrrhiza uralensis attenuating rheumatoid arthritis via reducing inflammation, suppressing angiogenesis, and inhibiting MAPK signaling pathway. J Agric Food Chem. 2019;67(10):2856-64. https://doi.org/10.1021/acs.jafc.9b00185.

47. Yang Y, Li S, Huang H, Lv J, Chen S, Pires Dias AC, et al. Comparison of the protective effects of ginsenosides $\mathrm{Rb} 1$ and Rg1 on improving cognitive deficits in SAMP8 mice based on anti-neuroinflammation mechanism. Front Pharmacol. 2020;11: 834. https://doi.org/10.3389/fphar.2020.00834.

48. Wang YS, Shen CY, Jiang JG. Antidepressant active ingredients from herbs and nutraceuticals used in TCM: pharmacological mechanisms and prospects for drug discovery. Pharmacol Res. 2019;150:104520. https://doi.org/10.1016/j.phrs.2019.104520.

49. Lim SI, Cho CW, Choi UK, Kim YC. Antioxidant activity and ginsenoside pattern of fermented white ginseng. J Ginseng Res. 2010;34(3):168-74. https://doi.org/10.5142/jgr.2010.34.3.168.

50. Yang N, Liang G, Lin J, Zhang S, Lin Q, Ji X, et al. Ginsenoside Rd therapy improves histological and functional recovery in a rat model of inflammatory bowel disease. Phytother Res. 2020;34(11): 3019-28. https://doi.org/10.1002/ptr.6734.

51. Filgueira MR, Huang Y, Witt K, Castells C, Carr PW. Improving peak capacity in fast online comprehensive two-dimensional liquid chromatography with post-first-dimension flow splitting. Anal Chem. 2011;83(24):9531-9. https://doi.org/10.1021/ac202317m.

52. Introduction. Comprehensive chromatography in combination with mass spectrometry. 2011. p. 1-11.

53. Neue UD. Theory of peak capacity in gradient elution. J Chromatogr A. 2005;1079(1-2):153-61. https://doi.org/10.1016/ j.chroma.2005.03.008.

54. An K, Jin-Rui G, Zhen Z, Xiao-Long W. Simultaneous quantification of ten active components in traditional Chinese formula Sijunzi decoction using a UPLC-PDA method. J Anal Methods Chem. 2014;2014:570359. https://doi.org/10.1155/2014/570359.

Publisher's note Springer Nature remains neutral with regard to jurisdictional claims in published maps and institutional affiliations. 